\title{
A Systematic Review of Factors Influencing Farmers' Adoption of Organic Farming
}

\author{
Ratana Sapbamrer ${ }^{1, *(D)}$ and Ajchamon Thammachai ${ }^{1,2}$ \\ 1 Department of Community Medicine, Faculty of Medicine, Chiang Mai University, Muang District, \\ Chiang Mai 50200, Thailand; ajchamon_thamma@cmu.ac.th \\ 2 Department of Physical Therapy, School of Allied Health Sciences, University of Phayao, Muang District, \\ Phayao 56000, Thailand \\ * Correspondence: ratana.sapbamrer@cmu.ac.th; Tel.: +66-53935-472
}

Citation: Sapbamrer, R.;

Thammachai, A. A Systematic Review of Factors Influencing Farmers' Adoption of Organic

Farming. Sustainability 2021, 13, 3842. https://doi.org/10.3390/su13073842

Academic Editor: Jan Moudrý

Received: 6 March 2021

Accepted: 23 March 2021

Published: 31 March 2021

Publisher's Note: MDPI stays neutral with regard to jurisdictional claims in published maps and institutional affiliations.

Copyright: (c) 2021 by the authors. Licensee MDPI, Basel, Switzerland. This article is an open access article distributed under the terms and conditions of the Creative Commons Attribution (CC BY) license (https:// creativecommons.org/licenses/by/ $4.0 /)$.

\begin{abstract}
Understanding the evidence-based factors that influence the adoption of organic farming yields benefits in terms of managing said adoption effectively. We searched relevant articles published in databases including Web of Science, Scopus, PubMed, Google Scholar, and Google. The inclusion criteria were as follows: original article; published in journals, proceedings, or dissertations; full article publication; published between 1999 and 2021; focusing on assessing the adoption of organic farming or conversion to organic farming; published in English; included data analyzed via correlation coefficient analysis, regression analysis, or discriminant analysis. Out of the 50 articles identified, 33 studies described findings associated with farmer and household factors, 32 described psychobehavioral and psychosocial factors, 36 assessed farming factors, and 33 analyzed supportive factors. The literature provides evidence that relevant factors include the following: (1) farmer and household factors (i.e., gender, off-farm income, and level of education); (2) psycho-behavioral and psychosocial factors (i.e., positive attitude, normative and moral obligations); (3) farming factors (i.e., organic farm experience, production cost, and farm ownership); (4) supportive factors (i.e., training, support of technology, organic farmer neighbors, information acquisition, membership of association, and extension contacts). To promote the adoption of organic farming, extension agents play a vital role in the provision of information via training and the encouragement of conventional farmers to shift towards organic agriculture. The target groups that have the greatest potential for the adoption of organic farming are young farmers, females, individuals who have ownership of their farm, those with a high level of education, and farmers with off-farm income. Farm associations also play a vital role in the sharing of experience and in increasing bargaining power. Support by the government in terms of resources, credit, markets, and subsidy is also relevant in motivating organic farming adoption. Therefore, three sectors, extension agents, farm associations, and the government, are key drivers for the sustainable adoption of organic farming.
\end{abstract}

Keywords: organic farming; farmers; determinant factors; adoption; conversion; sustainability

\section{Introduction}

During the last decade, concerns about the pesticide burden on the environment, ecosystem and health have been raised [1]. Pesticides have been shown to cause adverse health effects both in farmers and consumers. The adverse effects range from acute to chronic impacts, depending on type and level of pesticide exposure [2]. To address these problems, organic farming represents one of the most reasonable alternatives for sustainable agriculture $[3,4]$. Organic farming systems rely on biofertilizers, manure, crop rotation, and mechanical cultivation for the maintenance of soil productivity, enhancement of biodiversity, and the control of pests [4]. Global organic farmland increased to $20 \%$ of the total amount of farmland by the end of 2017. Furthermore, the proportion of organic farmland is increasing on all continents. Global organic markets have also increased 
worldwide, reaching USD 97 billion [5]. The acceptance of organic products by consumers has increased because they believe that organic products are healthier and more ecofriendly $[6,7]$.

Previous studies have discussed factors that influence the adoption of organic farming. Such studies have pointed out several factors, including demographic characteristics, psychobehavioral and psychosocial factors, farm structure, and other motivations. Most available studies have investigated the demographic characteristics and farming factors that influence the adoption of organic farming by farmers. However, the findings published in terms of the associations of some of these factors are inconsistent [8-10]. A study by Azam et al. [8] suggested that the age of farmers was more likely to have impact on the adoption of organic agriculture. Conversely, a study by Singh et al. [9] suggested that farmers' age was less likely to be a factor. Likewise, a study by Rana et al. [10] stated that farm size and farm experience were positively associated with the adoption of organic farming, whereas a study by Liu et al. [11] argued that farm size and farm experience were negatively associated with the adoption of organic farming. Although the available studies have investigated factors that influence the adoption of organic farming, the results of some factors were included or were not indicative of outcomes and a systematic analysis of the available literature has not been conducted.

It is known that organic farming is better for the environment, ecosystems, and individuals' health compared to conventional farming [4]. Therefore, understanding the evidence-based factors that influence the adoption of organic farming by farmers yields benefits in terms of effectively promoting the adoption of organic farming. A precise evidence-base would facilitate identifying target groups that could be encouraged to adopt organic farming. Therefore, here, a systematic review was conducted in order to assess the factors that influence the adoption of organic farming by farmers.

\section{Materials and Methods}

\subsection{Search Strategy}

We searched relevant articles published in databases including Web of Science, Scopus, PubMed, Google Scholar, and Google by using the following keywords: "factor" OR "determinant" OR "driver" plus "organic farming" OR "organic agriculture" and plus "adopt" OR "conversion". The review was conducted using the principles of the PRISMA (Preferred Reporting Items for Systematic Review and Meta-Analysis) framework.

\subsection{Inclusion Criteria}

We included articles that met the following characteristics: (1) original article; (2) published in journals, proceedings, or dissertations; (3) full article publication; (4) published between 1999 and 2021; (5) assessed the adoption of organic farming or conversion to organic farming; (6) published in English; (7) analyzed data via correlation coefficient analysis, regression analysis, or discriminant analysis to indicate the direction of association, with adjustment for confounding factors. We also excluded articles with the following characteristics: (1) articles that did not include variables of interest; (2) review articles; (3) articles with irrelevant information.

Figure 1 presents a flow diagram of the study selection procedures, which were as follows: 4621 articles were identified through database searches; 1185 articles remained after duplicate removal; 293 articles remained after screening of the abstract; 165 articles remained after screening full-text articles. This left 50 articles that were eligible for inclusion in the review. Out of the 50 articles, 33 studies described findings associated with farmer and household factors, 32 analyzed psychobehavioral and psychosocial factors, 36 discussed farming factors, and 33 assessed supportive factors. 
Records identified through database searches ( $\mathrm{n}=4621)$

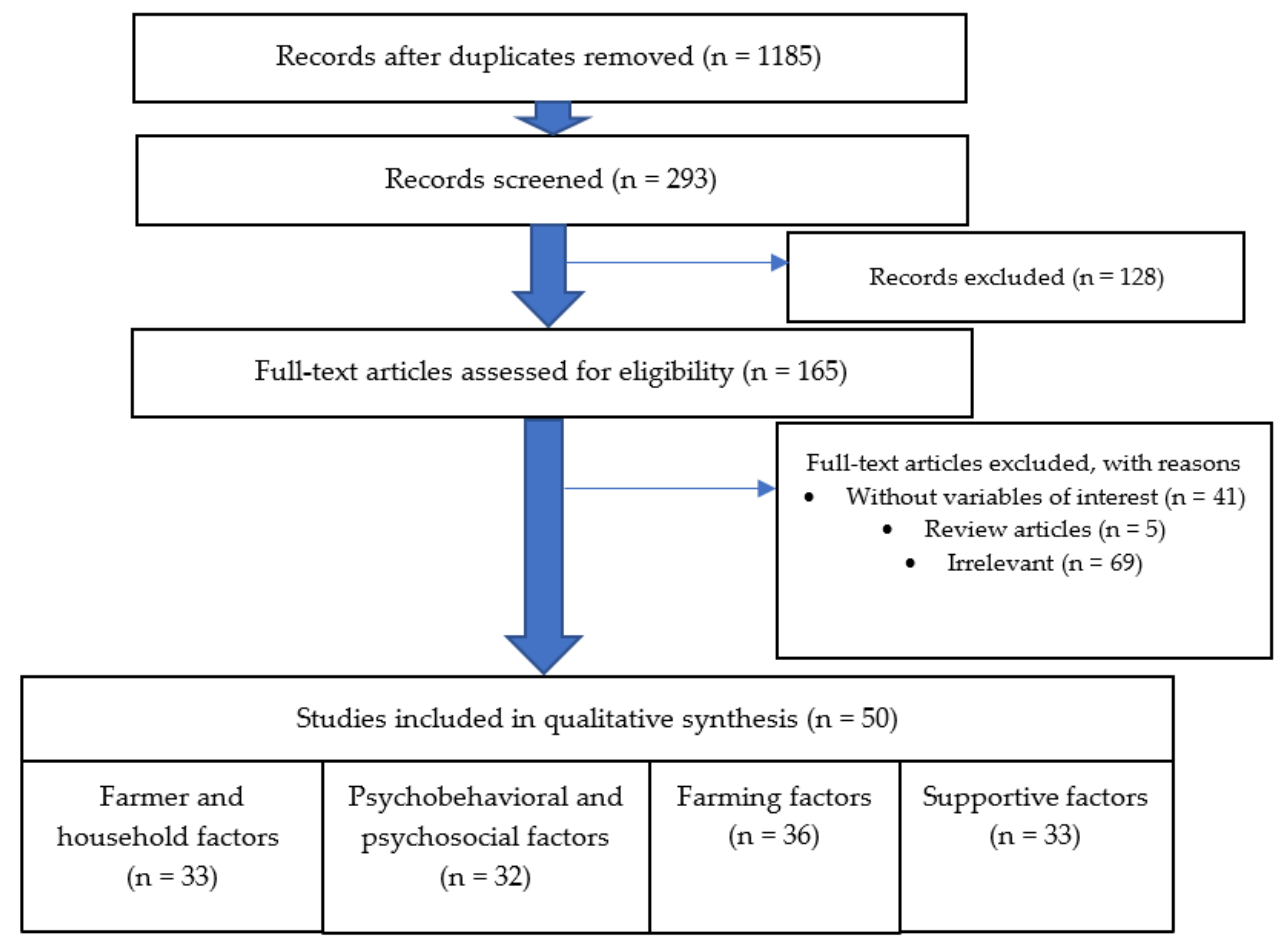

Figure 1. Flow chart of study selection.

\subsection{Data Analysis}

The data from the scientific literature were presented based on author, year of publication, country, study population, and findings. The literature was synthesized with regard to the factors influencing farmers' adoption of organic farming. The factors were classified into four groups of factors, including: (1) Farmer and household factors; (2) Psychobehavioral and psychosocial factors; (3) Farming factors; (4) Supportive factors. Factors with a positive association higher than $80 \%$ from the articles were concluded to be relevant factors influencing farmers' adoption of organic farming.

\section{Results and Discussion}

Table 1 shows the factors influencing farmers' adoption of organic farming, citing the 50 articles which were relevant. Table 2 summarizes the articles which describe the factors influencing farmers' adoption of organic farming.

\subsection{Farmer and Household Factors}

Farmer and household factors included age, education level, gender, marital status, income, off-farm job or income, household size, and others. 
Table 1. Factors influencing farmers' adoption of organic farming $(n=50)$.

\begin{tabular}{|c|c|c|c|c|}
\hline No. & Authors (Year) & Country & Population $(n)$ & Findings (Factors Associated with Adoption of Organic Farming) \\
\hline 1 & Egri (1999) [42] & Canada $^{a}$ & $\begin{array}{ll}203 & \\
- & 118 \text { organic } \\
- & 85 \text { conventional }\end{array}$ & $\begin{array}{ll}- & \text { Attitude towards organic farming }(\mathrm{B} \pm \mathrm{SE}=2.3902 \pm 0.6795, p<0.001) \\
\text { - } & \text { Attitude towards agrichemicals (environmental) }(\mathrm{B} \pm \mathrm{SE}=-1.2387 \pm 0.4906, p<0.05) \\
\text { - } & \text { Attitude towards agrichemicals (Economic) }(\mathrm{B} \pm \mathrm{SE}=-1.3776 \pm 0.5209, p<0.01)\end{array}$ \\
\hline 2 & Genius et al. (2003) [12] & Greece $^{b}$ & $\begin{array}{ll}237 & \\
\text { - } & 118 \text { non-adopters } \\
\text { - } \quad 75 \text { partial adopters } \\
\text { - } \quad 44 \text { full adopters }\end{array}$ & $\begin{array}{ll}- & \text { Age }\left(\mathrm{B}^{-}=-0.098\right) \\
- & \text { Farmers' education }(\mathrm{B}=0.237) \\
- & \text { Off-farm Income }(\mathrm{B}=0.023) \\
- & \text { Environmental awareness }(\mathrm{B}=0.041) \\
- & \text { Farm size }(\mathrm{B}=0.007) \\
- & \text { Farm specialization }(\mathrm{B}=-0.095) \\
- & \text { Aridity Index }(\mathrm{B}=-0.647) \\
- & \text { Active Information }(\mathrm{B}=0.190) \\
- & \text { Sxtension Contacts }(\mathrm{B}=0.103) \\
& \text { Subsidies }(\mathrm{B}=0.039)\end{array}$ \\
\hline 3 & $\begin{array}{l}\text { Hattam and Holloway (2005) } \\
{[55]}\end{array}$ & Mexico $^{b}$ & $\begin{array}{l}233 \\
-\quad 47 \text { organic } \\
\text { - } \quad 186 \text { conventional }\end{array}$ & $\begin{array}{ll}- & \text { Experience in agriculture }(\mathrm{B}=-0.029,95 \% \mathrm{CI}=-0.056,-0.003) \\
\text { - } & \text { Total cost per hectare }(\mathrm{B}=0.034,95 \% \mathrm{CI}=0.000,0.068) \\
\text { - } & \text { Make own inputs }(\mathrm{B}=1.774,95 \% \mathrm{CI}=1.061,2.517) \\
& \text { Membership of association }(\mathrm{B}=1.032,95 \% \mathrm{CI}=0.402,1.678)\end{array}$ \\
\hline 4 & $\begin{array}{l}\text { Parra López and Calatrava } \\
\text { Requena (2005) [38] }\end{array}$ & Spain ${ }^{a}$ & $\begin{array}{ll}322 & \\
\text { - } & 161 \text { organic } \\
\text { - } & 161 \text { conventional }\end{array}$ & $\begin{array}{ll}\text { - } & \text { Importance of agricultural activity in final income } \\
\text { - } & (\mathrm{B}+\mathrm{SE}=-1.940+0.613, p<0.01) \\
\text { - } & (\mathrm{B}+\mathrm{SE}=1.970+0.505, p<0.01) \\
\text { - } & \text { Opinion on the economic profitability of organic farming } \\
\text { - } & (\mathrm{B}+\mathrm{SE}=-1.585+0.488, p<0.01) \\
\text { - } & \text { Opinion on the time and effort saving of organic agriculture } \\
\text { - } & (\mathrm{B}+\mathrm{SE}=2.014+0.525, p<0.01) \\
\text { - } & \text { First knowledge of organic farming }(\mathrm{B}+\mathrm{SE}=3.242+0.910, p<0.01) \\
\text { - } & \text { Years dedicated to agriculture }(\mathrm{B}+\mathrm{SE}=-0.022+0.010, p<0.05) \\
\text { - } & \text { Productivity of the plantation }(\mathrm{B}+\mathrm{SE}=-0.001+0.000, p<0.01) \\
\text { - Type of work carried out directly on the farm }(\mathrm{B}+\mathrm{SE}=-3.066+1.387, p<0.05) \\
\text { - } & \text { Agricultural training }(\mathrm{B}+\mathrm{SE}=1.105+0.486, p<0.05) \\
\text { - } & \text { Membership of Integrated Pest Management }(\mathrm{B}+\mathrm{SE}=2.157+0.448, p<0.01)\end{array}$ \\
\hline
\end{tabular}


Table 1. Cont.

\begin{tabular}{|c|c|c|c|c|}
\hline No. & Authors (Year) & Country & Population $(n)$ & Findings (Factors Associated with Adoption of Organic Farming) \\
\hline 5 & Anderson et al. (2005) [13] & California, USA ${ }^{a}$ & $\begin{array}{l}175 \\
\text { - } \quad 28 \text { organic } \\
\text { - } \quad 118 \text { conventional } \\
\text { - } 29 \text { mixed }\end{array}$ & $\begin{array}{ll}- & \text { Age }(\mathrm{B}+\mathrm{SE}=-0.097+0.035, p<0.01) \\
- & \text { Total number of crops grown per farm }(\mathrm{B}+\mathrm{SE}=0.129+0.065, p<0.05) \\
- & \text { Direct marketing }(\mathrm{B}+\mathrm{SE}=1.645+0.616, p<0.01) \\
- & \text { Use of computers in production management }(\mathrm{B}+\mathrm{SE}=2.242+0.697, p<0.01)\end{array}$ \\
\hline 6 & De Cock (2005) [43] & Belgium ${ }^{\mathrm{f}}$ & $\begin{array}{l}283 \\
\text { - } \quad 93 \text { organic } \\
190 \text { non-organic }\end{array}$ & $\begin{array}{ll}- & \text { Attitude }(\mathrm{B}+\mathrm{SE}=0.764+0.239, p<0.01) \\
- & \text { Perceived feasibility }(\mathrm{B}+\mathrm{SE}=0.486+0.130, p<0.001) \\
- & \text { Concern in quality and environment }(\mathrm{B}+\mathrm{SE}=-0.579+0.230, p<0.05) \\
- & \text { Social pressure }(\mathrm{B}+\mathrm{SE}=0.961+0.406, p<0.05) \\
- & \text { Intension of information search }(\mathrm{B}+\mathrm{SE}=0.439+0.097, p<0.001)\end{array}$ \\
\hline 7 & Isin et al. (2007) [14] & Turkey $^{\mathrm{b}}$ & $\begin{array}{l}127 \\
\bullet \quad 20 \text { organic } \\
-\quad 107 \text { traditional }\end{array}$ & $\begin{array}{ll}- & \text { Age }(\mathrm{B}+\mathrm{SE}=-0.0716+0.0304, p<0.05) \\
\text { - } & \text { Education }(\mathrm{B}+\mathrm{SE}=0.2542+0.1073, p<0.05) \\
\text { - } & \text { Experience }(\mathrm{B}+\mathrm{SE}=0.0601+0.0281, p<0.05) \\
\text { - } & \text { Total production }\left(\mathrm{B}+\mathrm{SE}=0.0001+5.24 \times 10^{-5}, p<0.05\right)\end{array}$ \\
\hline 9 & McCarthy et al. (2007) [44] & Ireland $^{\mathrm{a}}$ & - $\quad 252$ organic & $\begin{array}{ll}- & \text { Attitude }(\mathrm{B}=0.233, p<0.001) \\
- & \text { Subjective norm }(\mathrm{B}=0.114, p<0.05) \\
- & \text { Moral obligation }(\mathrm{B}=0.165, p<0.01) \\
\text { - } & \text { Self-identity }(\mathrm{B}=0.328, p<0.001)\end{array}$ \\
\hline 10 & Canavari et al. (2008) [53] & Italy $^{a}$ & $\begin{array}{l}332 \\
\text { (organic, conventional and } \\
\text { mixed producers) }\end{array}$ & $\begin{array}{l}\text { - } \quad \text { Attitude towards "Disagree about suitability of the territory towards organic practices" } \\
\text { - } \mathrm{B}+\mathrm{SE}=-2.561+0.887, p<0.01) \\
\text { - } \quad \text { Problems related to the certification system }(\mathrm{B}+\mathrm{SE}=-0.498+0.203, p<0.05) \\
\text { Farm typology (Mixed) }(\mathrm{B}+\mathrm{SE}=1.599+0.739, p<0.05)\end{array}$ \\
\hline
\end{tabular}


Table 1. Cont.

\begin{tabular}{|c|c|c|c|c|}
\hline No. & Authors (Year) & Country & Population $(n)$ & Findings (Factors Associated with Adoption of Organic Farming) \\
\hline 11 & Koesling et al. (2008) [25] & Norway ${ }^{a}$ & $\begin{array}{l}1018 \\
-\quad 297 \text { organic } \\
\text { - } \quad 721 \text { conventional }\end{array}$ & $\begin{array}{ll}\text { - } & \text { Agricultural education }(\mathrm{RRR}=3.296, p<0.001) \\
\text { - } & \text { Reliable and stable income }(\mathrm{RRR}=0.317, p<0.001) \\
\text { - } & \text { Attitude about "Use of pesticides decreases food quality" } \\
\text { - } & (\mathrm{RRR}=1.404, p<0.001) \\
\text { - } & \text { Attitude towards "Organic livestock farming takes more consideration to animals' } \\
\text { - } & \text { Atural requirements" (RRR }=1.257, p<0.01) \\
\text { - } & \text { (RRR }=0.477, p<0.001 \\
\text { - } & \text { Attitude towards "Conventional farming is more sustainable than organic" (RRR }= \\
\text { - } & \text { Attitude } p<0.001) \\
\text { - } & \text { (RRR }=0.709, p<0.001) \\
\text { - } & \text { Location }(\mathrm{RRR}=2.074, p<0.05)\end{array}$ \\
\hline 12 & Peter Silas (2008) [40] & Kenya $^{a}$ & $\begin{array}{l}119 \\
\text { - } \quad 56 \text { adopters } \\
\text { - } \quad 63 \text { non-adopters }\end{array}$ & $\begin{array}{ll}- & \text { Household size }(\mathrm{B}=0.709, p<0.01) \\
- & \text { Farm Size }(\mathrm{B}=0.905, p<0.01) \\
- & \text { Membership of organic farming }(\mathrm{B}=3.389, p<0.01) \\
- & \text { Eco-zones }(\mathrm{B}=2.809, p<0.05)\end{array}$ \\
\hline 13 & Alexopoulos et al. (2010) [24] & Western Greece ${ }^{b}$ & $\begin{array}{ll}364 & \\
\bullet & 187 \text { organic } \\
- & 177 \text { conventional }\end{array}$ & $\begin{array}{l}\text { - } \quad \text { Other occupation }(\mathrm{B}+\mathrm{SE}=1.2693+0.5586, p<0.05) \\
\text { "People who they trust believe that } \mathrm{I} \text { have to follow organic agriculture" }(\mathrm{B}+\mathrm{SE}= \\
-4.5926+1.1270, p<0.01) \\
\text { - } \quad \text { Farm size }(\mathrm{B}+\mathrm{SE}=0.0146+0.0065, p<0.05) \\
\text { - }\end{array}$ \\
\hline 14 & Best (2010) [57] & Western Germany ${ }^{a}$ & $\begin{array}{l}\text { 657 } \\
\text { - } \quad 494 \text { organic } \\
\text { - } \quad 163 \text { conventional }\end{array}$ & $\begin{array}{ll}- & \text { Opinion colleagues }(\mathrm{B}=0.29, p<0.001) \\
- & \text { Opinion family }(\mathrm{B}=0.29, p<0.001) \\
- & \text { Fodder crop/cattle husbandry }(\mathrm{B}=0.24, p<0.001) \\
- & \text { Pig/Poultry husbandry }(\mathrm{B}=0.19, p<0.01) \\
- & \text { Other husbandry }(\mathrm{B}=0.21, p<0.05) \\
- & \text { Utility difference between conventional and organic farming } \\
- & (\mathrm{B}=0.36, p<0.001)\end{array}$ \\
\hline
\end{tabular}


Table 1. Cont.

\begin{tabular}{|c|c|c|c|c|}
\hline No. & Authors (Year) & Country & Population $(n)$ & Findings (Factors Associated with Adoption of Organic Farming) \\
\hline 15 & Khaledi et al. (2010) [15] & Canada ${ }^{\mathrm{f}}$ & $\begin{array}{l}57 \quad 27 \text { complete organic } \\
\text { - } \quad 30 \text { partial organic }\end{array}$ & $\begin{array}{ll}- & \text { Age }(\mathrm{B}=-0.4977, p<0.05) \\
- & \text { Hourly wage paid to self }(\mathrm{B}=-0.0176, p<0.01) \\
- & \text { Total cultivated area }(\mathrm{B}=-0.0003, p<0.01) \\
- & \text { Average satisfaction with marketer functions }(\mathrm{B}=0.0517, p<0.01) \\
- & \text { Internet use in marketing organic products }(\mathrm{B}=0.5169, p<0.01) \\
- & \text { Marketing problems rank }(\mathrm{B}=-0.3165, p<0.01) \\
- & \text { Distance to cleaning location }(\mathrm{B}=-0.0031, p<0.05)\end{array}$ \\
\hline 16 & Läpple (2010) [45] & Ireland $^{\text {a }}$ & $\begin{array}{ll}546 & \\
\text { - } & 341 \text { organic } \\
\text { - } & 41 \text { ex-organic } \\
\text { - } & 164 \text { conventional }\end{array}$ & $\begin{array}{ll}- & \text { Farming experience }(\mathrm{B}+\mathrm{SE}=0.02+0.01, p<0.01) \\
- & \text { Environmental attitude }(\mathrm{B}+\mathrm{SE}=0.81+0.08, p<0.01) \\
- & \text { Risk attitude }(\mathrm{B}+\mathrm{SE}=-0.11+0.05, p<0.05) \\
- & \text { Utilization agricultural area }(\mathrm{B}+\mathrm{SE}=-0.01+0.00, p<0.01) \\
- & \text { Single farm payment }(\mathrm{B}+\mathrm{SE}=0.73+0.12, p<0.01) \\
- & \text { Livestock unit per hectare }(\mathrm{B}+\mathrm{SE}=-0.38+0.14, p<0.05) \\
- & \text { Cattle }(\mathrm{B}+\mathrm{SE}=-0.04+0.00, p<0.01) \\
- & \text { Knows another organic farmer }(\mathrm{B}+\mathrm{SE}=0.82+0.16, p<0.01)\end{array}$ \\
\hline 18 & Karki et al. (2011) [21] & Nepal $^{\mathrm{d}}$ & $\begin{array}{ll}181 & \\
- & 86 \text { organic } \\
\text { - } & 95 \text { conventional }\end{array}$ & $\begin{array}{ll}- & \text { Age }(\mathrm{LDF}=5.906, p<0.01) \\
- & \text { Ethnicity }(\mathrm{LDF}=13.723, p<0.01) \\
\text { - } & \text { Farm size }(\mathrm{LDF}=55.247, p<0.01) \\
\text { - } & \text { Participation in training }(\mathrm{LDF}=18.042, p<0.01) \\
\text { - } & \text { Affiliation with institutions }(\mathrm{LDF}=167.831, p<0.01) \\
\text { - } & \text { Time required to reach the market }(\mathrm{LDF}=189.485, p<0.01)\end{array}$ \\
\hline 19 & Kafle (2011) [54] & Nepal $^{c}$ & $\begin{array}{l}65 \text { (complete } \\
\text { adopters/partial adopters) }\end{array}$ & $\begin{array}{ll}- & \text { Farm size }(B=0.345, p<0.05) \\
- & \text { Participation in training and visits }(B=0.431, p<0.01) \\
- & \text { Compatibility to organic farming }(B=0.320, p<0.05)\end{array}$ \\
\hline
\end{tabular}



Table 1. Cont.

\begin{abstract}
No.
\end{abstract}
Authors (Year)

Country

Population (n)

Findings (Factors Associated with Adoption of Organic Farming)

218
- 82 certified organic

- 136 being in-conversion

- Attitude towards "Influence of organic farming to manage a farm effectively" (B + SE $=1.196+0.261, p<0.001)$

- Attitude towards "Organic farming increases the value of the land" (B + SE $=0.969+$ $0.235, p<0.001)$

- $\quad$ Farm type $(\mathrm{B}+\mathrm{SE}=1.599+0.685, p<0.05)$

- Number of agricultural information sources used

- $\quad(\mathrm{B}+\mathrm{SE}=0.635+0.184, p<0.001)$

- Number of organic farmers with whom regularly discussed

- $\quad(\mathrm{B}+\mathrm{SE}=1.026+0.182, p<0.001)$

- Pioneer:

- $\quad$ Age $(\mathrm{RRR}=0.85, \mathrm{SE}=0.03, p<0.001)$

- $\quad$ Household members $(\mathrm{RRR}=0.67, \mathrm{SE}=0.13, p<0.05)$

- Environmental attitude $(\mathrm{RRR}=26.08, \mathrm{SE}=12.35, p<0.001)$

- Utilizable agricultural area $(\mathrm{RRR}=0.98, \mathrm{SE}=0.008, p<0.05)$

- $\quad$ Profit orientation $(\mathrm{RRR}=0.48, \mathrm{SE}=0.14, p<0.05)$

- $\quad$ Livestock unit $(\mathrm{RRR}=0.24, \mathrm{SE}=0.16, p<0.05)$

- $\quad$ Frequency of information $(\mathrm{RRR}=0.66, \mathrm{SE}=0.13, p<0.05)$

- $\quad$ Knows other organic farmer $(\mathrm{RRR}=9.74, \mathrm{SE}=7.63, p<0.001)$

- Followers:

- $\quad$ Age $(\mathrm{RRR}=0.91, \mathrm{SE}=0.02, p<0.001)$

- 164 non-adopters

$21 \quad$ Läpple and Rensburg (2011) [16]

Ireland $^{a}$

- 29 pioneers

- 216 followers

- 137 laggards

- Environmental attitude $(\mathrm{RRR}=21.42, \mathrm{SE}=7.40, p<0.001)$

- $\quad$ Risk attitude $(\mathrm{RRR}=0.59, \mathrm{SE}=0.14, p<0.05)$

- $\quad$ Utilizable agricultural area $(\mathrm{RRR}=0.97, \mathrm{SE}=0.006, p<0.001)$

- $\quad$ Frequency of information $(\mathrm{RRR}=0.66, \mathrm{SE}=0.09, p<0.001)$

- $\quad$ Knows other organic farmer $(\mathrm{RRR}=8.41, \mathrm{SE}=3.90, p<0.001)$

- Laggards:

- $\quad$ Age $(\mathrm{RRR}=0.94, \mathrm{SE}=0.02, p<0.001)$

- $\quad$ Environmental attitude $(\mathrm{RRR}=15.25, \mathrm{SE}=5.25, p<0.001)$

- $\quad$ Risk attitude $(\mathrm{RRR}=0.60, \mathrm{SE}=0.15, p<0.05)$

- $\quad$ Utilizable agricultural area $(\mathrm{RRR}=0.97, \mathrm{SE}=0.007, p<0.001)$

- $\quad$ Frequency of information $(\mathrm{RRR}=0.66, \mathrm{SE}=0.09, p<0.001)$

- Frequency of consultation with a farm advisor $(\mathrm{RRR}=1.99, \mathrm{SE}=0.62, p<0.05)$

- $\quad$ Knows other organic farmer $(\mathrm{RRR}=8.40, \mathrm{SE}=3.99, p<0.001)$ 
Table 1. Cont.

\begin{tabular}{|c|c|c|c|c|}
\hline No. & Authors (Year) & Country & Population $(n)$ & Findings (Factors Associated with Adoption of Organic Farming) \\
\hline 22 & Mzoughi (2011) [47] & France $^{a}$ & $\begin{array}{ll}243 & \\
- & 38 \text { organic } \\
- & 71 \text { integrate } \\
- & 134 \text { conventional }\end{array}$ & $\begin{array}{l}\text { - } \quad \text { Social concern about environmental commitment to others } \\
\text { - } \quad(\mathrm{B}=1.182, p<0.05) \\
\text { - } \quad \text { Economic concern about cutting production cost }(\mathrm{B}=-1.540, p<0.05) \\
\quad \text { Economic concern about risk reduction }(\mathrm{B}=-1.664, p<0.01)\end{array}$ \\
\hline 23 & $\begin{array}{l}\text { Pornpratansombat et al. } \\
\text { (2011) [48] }\end{array}$ & Thailand $^{\text {a }}$ & $\begin{array}{l}\text { - } 90 \text { organic } \\
\text { - } \quad 90 \text { conventional }\end{array}$ & $\begin{array}{ll}\text { - } & \text { Attitude }- \text { towards “Conventional technology induces on }- \text { farm problem" }(\mathrm{HR}=1.589, \\
\mathrm{SE}=0.224, p<0.01) \\
\text { - } \quad \text { Farm }- \text { gate price }(\mathrm{HR}=1.415, \mathrm{SE}=0.092, p<0.001) \\
\text { - } \text { Access water during dry period }(\mathrm{HR}=2.249, \mathrm{SE}=0.607, p<0.01)\end{array}$ \\
\hline 24 & Radwan et al. (2011) [26] & Egypt $^{\mathrm{a}}$ & 60 (organic/non-organic) & $\begin{array}{ll}- & \text { Education }(\mathrm{HR}=1.52, p<0.05) \\
\text { - } & \text { Perceived risk (risk that is willing to undertake) }(\mathrm{HR}=1.34, p<0.001)\end{array}$ \\
\hline 25 & Saoke (2011) [17] & Kenya $^{a}$ & 80 farmers & $\begin{array}{ll}- & \text { Age }(\mathrm{B}+\mathrm{SE}=-0.085+0.003, p<0.05) \\
- & \text { Years in organic farming }(\mathrm{B}+\mathrm{SE}=0.140+0.005, p<0.05) \\
- & \text { Others in organic farming }(\mathrm{B}+\mathrm{SE}=-1.743+0.043, p<0.05) \\
- & \text { Member of farmer group }(\mathrm{B}+\mathrm{SE}=-2.126+0.115, p<0.05) \\
& \text { Access to credit }(\mathrm{B}+\mathrm{SE}=-2.326+0.282, p<0.05)\end{array}$ \\
\hline 26 & $\begin{array}{l}\text { Prashanth and Reddy (2012) } \\
\text { [27] }\end{array}$ & India ${ }^{e}$ & $\begin{array}{ll}120 & \\
- & 60 \text { organic } \\
- & 60 \text { conventional }\end{array}$ & $\begin{array}{ll}- & \text { Education }(\mathrm{r}=0.211, p<0.05) \\
- & \text { Farm size }(\mathrm{r}=-0.285, p<0.05) \\
- & \text { Herd size }(\mathrm{r}=0.392, p<0.01) \\
- & \text { Training received }(\mathrm{r}=0.279, p<0.05) \\
- & \text { Extension contact }(\mathrm{r}=0.420, p<0.01) \\
- & \text { Organic inputs utilization pattern }(\mathrm{r}=0.238, p<0.05) \\
- & \text { Decision making behavior }(\mathrm{r}=0.209, p<0.05)\end{array}$ \\
\hline 27 & Adesope et al. (2012) [20] & Nigeria ${ }^{e}$ & 90 farmers & $\begin{array}{ll}\text { - } & \text { Marital status }(\mathrm{r}=-0.221, p<0.05) \\
\text { - } & \text { Farming experience }(\mathrm{r}=-0.277, p<0.01)\end{array}$ \\
\hline
\end{tabular}


Table 1. Cont.

\begin{tabular}{|c|c|c|c|c|}
\hline No. & Authors (Year) & Country & Population $(n)$ & Findings (Factors Associated with Adoption of Organic Farming) \\
\hline 28 & Rana et al. (2012) [10] & India $^{\text {a }}$ & $\begin{array}{ll} & \\
\bullet & 100 \text { organic } \\
- & 100 \text { conventional }\end{array}$ & $\begin{array}{ll}- & \text { Age }(\mathrm{B}=-0.071, p<0.05) \\
& \text { Total agricultural area }(\mathrm{B}=0.719, p<0.01) \\
- & \text { Farming experience }(\mathrm{B}=0.085, p<0.01) \\
- & \text { Extension service }(\mathrm{B}=-1.572, p<0.01) \\
& \text { Access to credit }(\mathrm{B}=1.954, p<0.01)\end{array}$ \\
\hline 29 & Savari et al. (2013) [49] & $\operatorname{Iran}^{\mathrm{a}}$ & 155 farmers & $\begin{array}{ll}- & \text { Attitude towards organic farming }(B=1.125, p<0.001) \\
\text { - } & \text { Communicative channels and resources }(B=3.011, p<0.001) \\
\quad & \text { Application of educational publications }(B=1.236, p<0.001)\end{array}$ \\
\hline 30 & Malá and Malý (2013) [18] & Czech Republic ${ }^{\text {a }}$ & 531 agricultural business & $\begin{array}{ll}- & \text { Age }(\mathrm{B}+\mathrm{SE}=-0.2876+0.1045, p<0.01) \\
- & \text { Gender }(\mathrm{Female})(\mathrm{B}+\mathrm{SE}=0.3943+0.1511, p<0.01) \\
- & \text { Farm size }(\mathrm{B}+\mathrm{SE}=-0.4660+0.0631, p<0.01) \\
- & \text { Location (South East region) }(\mathrm{B}+\mathrm{SE}=2.2461+0.6444, p<0.01) \\
- & \text { Location (North East region) }(\mathrm{B}+\mathrm{SE}=1.7380+0.6532, p<0.01) \\
& \text { Location (North West region) }(\mathrm{B}+\mathrm{SE}=2.7385+0.6660, p<0.01) \\
- & \text { Location (Central Moravia region) }(\mathrm{B}+\mathrm{SE}=2.5852+0.6431, p<0.01) \\
- & \text { Location (Moravia-Silesia region) }(\mathrm{B}+\mathrm{SE}=2.5705+0.6647, p<0.01) \\
& \text { Profitable of cost }(\mathrm{B}+\mathrm{SE}=4.2109+0.5549, p<0.01) \\
& \text { Mixed production }(\mathrm{B}+\mathrm{SE}=-0.8050+0.2331, p<0.01) \\
- & \text { Average rate of organic farming subsidy }(\mathrm{B}+\mathrm{SE}=1.000+0.3355, p<0.01) \\
- & \text { Age of farm }(\mathrm{B}+\mathrm{SE}=1.5987+0.1792, p<0.01) \\
& \text { Labor productivity }(\mathrm{B}+\mathrm{SE}=-1.0648+0.1832, p<0.01)\end{array}$ \\
\hline 31 & $\begin{array}{l}\text { Wollni and Andersson (2014) } \\
\text { [22] }\end{array}$ & Honduras ${ }^{b}$ & $\begin{array}{ll}239 \\
-\quad 47 \text { organic } \\
-\quad 192 \text { conventional }\end{array}$ & 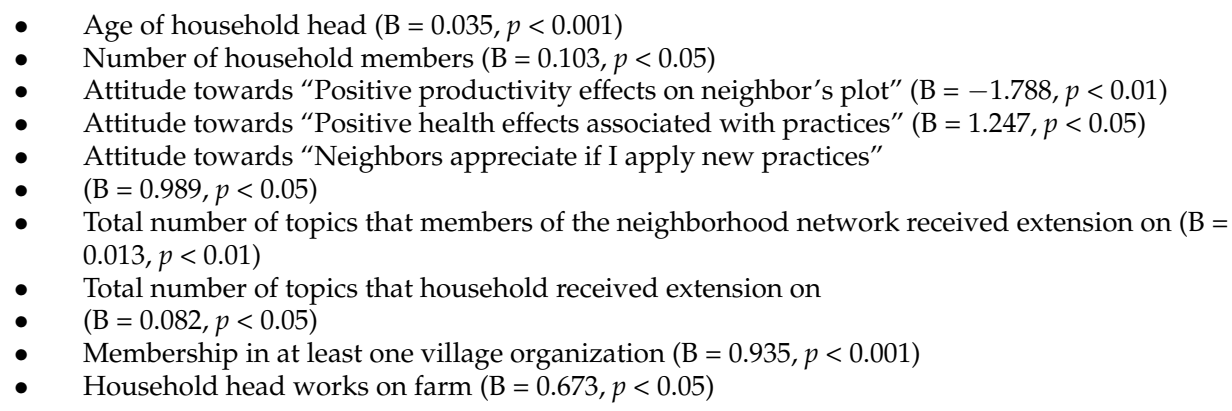 \\
\hline
\end{tabular}


Table 1. Cont.

\begin{tabular}{|c|c|c|c|c|}
\hline No. & Authors (Year) & Country & Population $(n)$ & Findings (Factors Associated with Adoption of Organic Farming) \\
\hline 32 & $\begin{array}{l}\text { Sriwichailamphan and } \\
\text { Sucharidtham (2014) [35] }\end{array}$ & Thailand $^{\text {a }}$ & $\begin{array}{l}600 \\
-\quad 304 \text { organic } \\
\text { - } \quad 296 \text { non-organic }\end{array}$ & $\begin{array}{ll}- & \text { Gender (male) }(\mathrm{B}+\mathrm{SE}=-0.250+0.126, p<0.05) \\
- & \text { Income from secondary occupation }(\mathrm{B}+\mathrm{SE}=0.220+0.096, p<0.05) \\
- & \text { Ethnicity }(\mathrm{Hmong})(\mathrm{B}+\mathrm{SE}=-0.468+0.079, p<0.01) \\
- & \text { Awareness of beneficial effect }(\mathrm{B}+\mathrm{SE}=0.337+0.107, p<0.01) \\
- & \text { Concern to save production cost }(\mathrm{B}+\mathrm{SE}=0.365+0.121, p<0.01) \\
& \text { Perceive that organic farming must be isolated from farming areas where } \\
& \text { agrochemicals were applied }(\mathrm{B}+\mathrm{SE}=0.318+0.128, p<0.05) \\
- & \text { Farm size }(\mathrm{B}+\mathrm{SE}=-0.096+0.022, p<0.01) \\
- & \text { Participation in training }(\mathrm{B}+\mathrm{SE}=0.500+0.088, p<0.01) \\
- & \text { Frequency of training }(\mathrm{B}+\mathrm{SE}=0.144+0.045, p<0.01) \\
- & \text { Join the organic farming }(\mathrm{B}+\mathrm{SE}=0.506+0.085, p<0.01) \\
- & \text { Service soil analysis every year }(\mathrm{B}+\mathrm{SE}=0.288+0.097, p<0.01)\end{array}$ \\
\hline 33 & Shaban (2015) [28] & Gaza Strip ${ }^{a}$ & $\begin{array}{ll}100 & \\
\text { - } \quad & 70 \text { accepting organic } \\
\text { - } \quad 30 \text { rejecting organic }\end{array}$ & $\begin{array}{ll}- & \text { Education of farmer }(\mathrm{B}+\mathrm{SE}=1.59+0.57, p<0.01) \\
- & \text { Attitude towards organic farming }(\mathrm{B}+\mathrm{SE}=3.962+1.203, p<0.01)\end{array}$ \\
\hline 35 & Pinthukas (2015) [29] & Thailand $^{\mathrm{c}}$ & 108 organic & $\begin{array}{ll}- & \text { Education }(\mathrm{B}+\mathrm{SE}=3.231+1.338, p<0.05) \\
- & \text { Experience }(\mathrm{B}+\mathrm{SE}=-4.756+1.245, p<0.001) \\
- & \text { Access to natural water }(\mathrm{B}+\mathrm{SE}=-9.152+3.250, p<0.01)\end{array}$ \\
\hline 36 & Sodjinou et al. (2015) [32] & West Africa ${ }^{b}$ & 81 farmers & $\begin{array}{ll}- & \text { Formal education }(\mathrm{B}+\mathrm{SE}=-0.118+0.053, p<0.05) \\
- & \text { Gender (male) }(\mathrm{B}+\mathrm{SE}=-1.542+0.441, p<0.01) \\
- & \text { Regional dummy }(\mathrm{B}+\mathrm{SE}=1.346+0.641, p<0.05) \\
- & \text { Distance between house and farm }(\mathrm{B}+\mathrm{SE}=-1.112+0.326, p<0.01) \\
- & \text { Contact with extension agents }(\mathrm{B}+\mathrm{SE}=0.355+0.073, p<0.01)\end{array}$ \\
\hline
\end{tabular}


Table 1. Cont.

\begin{tabular}{|c|c|c|c|c|}
\hline No. & Authors (Year) & Country & Population $(n)$ & Findings (Factors Associated with Adoption of Organic Farming) \\
\hline 37 & Ullah et al. (2015) [41] & Pakistan $^{a}$ & 100 farmers & - $\quad$ Attitude-adverse effect of inorganic fertilizer $(\mathrm{B}+\mathrm{SE}=0.843+0.43, p<0.05)$ \\
\hline 38 & Xie et al. (2015) [23] & China $^{a}$ & 140 farmers & $\begin{array}{ll}- & \text { Age }(\mathrm{B}+\mathrm{SE}=0.588+0.264, p<0.05) \\
- & \text { Perceive risk (Risk preference) }(\mathrm{B}+\mathrm{SE}=0.645+0.160, p<0.01) \\
\quad & \text { Perceived benefit in income (increase revenue })(\mathrm{B}+\mathrm{SE}=2.769+0.527, p<0.01) \\
& \text { Adequate of labor }(\mathrm{B}+\mathrm{SE}=1.272+0.547, p<0.05)\end{array}$ \\
\hline 39 & Azam and Banumathi (2015) [8] & India $^{\text {a }}$ & $\begin{array}{ll}160 & \\
-\quad 80 \text { organic } \\
& 80 \text { conventional }\end{array}$ & $\begin{array}{ll}- & \text { Age }(\mathrm{B}+\mathrm{SE}=0.897+0.372, p<0.05) \\
- & \text { Education }(\mathrm{B}+\mathrm{SE}=0.517+0.211, p<0.05) \\
\text { - } & \text { Gender }(\mathrm{female})(\mathrm{B}+\mathrm{SE}=1.536+0.642, p<0.05) \\
& \text { Marital status }(\mathrm{B}+\mathrm{SE}=-1.875+1.011, p<0.10) \\
& \text { Family type }(\mathrm{B}+\mathrm{SE}=-0.999+0.478, p<0.05)\end{array}$ \\
\hline 40 & Cukur (2015) [33] & Turkey $^{\mathrm{b}}$ & 71 farmers & $\begin{array}{ll}- & \text { Education }(\mathrm{B}+\mathrm{SE}=-3.223867+1.576727, p<0.05) \\
\quad \text { Information about organic agriculture }(\mathrm{B}+\mathrm{SE}=3.563944+1.327854, p<0.01)\end{array}$ \\
\hline 41 & Kerdsriserm et al. (2016) [36] & Thailand $^{\mathrm{c}}$ & 30 organic & $\begin{array}{ll}- & \text { Gender (female) }(\mathrm{B}+\mathrm{SE}=0.904+0.413, p<0.05) \\
\text { - } & \text { Participation in training }(\mathrm{B}+\mathrm{SE}=0.678+0.280, p<0.05)\end{array}$ \\
\hline 43 & Pradhan et al. (2017) [31] & India ${ }^{e}$ & 90 farmers & $\begin{array}{ll}- & \text { Education }(\mathrm{r}=0.257, p<0.05) \\
- & \text { Annual income }(\mathrm{r}=0.221, p<0.05) \\
- & \text { Farm size }(\mathrm{r}=-0.233, p<0.05) \\
: & \text { Organic farming experience }(\mathrm{r}=0.238, p<0.05) \\
- & \text { Institutional approach towards promotion of organic farming } \\
& (\mathrm{r}=0.288, p<0.01) \\
& \text { Use of mass media }(\mathrm{r}=0.229, p<0.05) \\
& \text { Innovation proneness }(\mathrm{r}=0.297, p<0.01)\end{array}$ \\
\hline
\end{tabular}


Table 1. Cont.

\begin{tabular}{|c|c|c|c|c|}
\hline No. & Authors (Year) & Country & Population $(n)$ & Findings (Factors Associated with Adoption of Organic Farming) \\
\hline 44 & Haris et al. (2018) [50] & Malaysia $^{\text {a }}$ & $\begin{array}{ll}170 & \\
- & 82 \text { organic } \\
- & 88 \text { conventional }\end{array}$ & $\begin{array}{ll}- & \text { Environmental attitude }(\mathrm{B}+\mathrm{SE}=2.730+0.828, p<0.01) \\
- & \text { Profitable attitude }(\mathrm{B}+\mathrm{SE}=-2.817+0.843, p<0.01) \\
- & \text { Risk averse attitude }(\mathrm{B}+\mathrm{SE}=-1.775+0.729, p<0.05) \\
- & \text { Farm ownership }(\mathrm{B}+\mathrm{SE}=1.620+0.727, p<0.05) \\
& \text { Training }(\mathrm{B}+\mathrm{SE}=1.595+0.733, p<0.05) \\
- & \text { Information seek attitude }(\mathrm{B}+\mathrm{SE}=1.799+0.893, p<0.05) \\
- & \text { Membership farmers organization }(\mathrm{B}+\mathrm{SE}=-1.831+0.630, p<0.01) \\
- & \text { Business motivation and environmental attitude }(\mathrm{B}+\mathrm{SE}=1.562+0.435, p<0.001) \\
& \text { Organic Lifestyle }(\mathrm{B}+\mathrm{SE}=1.510+0.530, p<0.01) \\
& \text { Others influence }(\mathrm{B}+\mathrm{SE}=-0.777+0.395, p<0.05)\end{array}$ \\
\hline 45 & Métouolé Méda et al. (2018) [19] & Ghana $^{a}$ & $\begin{array}{ll} & \\
\bullet & 55 \text { organic } \\
- & 152 \text { conventional } \\
- & 177 \mathrm{GM}\end{array}$ & $\begin{array}{l}\text { Education }(\mathrm{B}+\mathrm{SE}=4.792+2.234, p<0.05) \\
\text { - } \quad \text { Gender }(\mathrm{female})(\mathrm{B}+\mathrm{SE}=6.090+2.759, p<0.05) \\
\text { Farm size }(\mathrm{B}+\mathrm{SE}=-7.585+2.631, p<0.01) \\
\text { Location }(\mathrm{B}+\mathrm{SE}=5.152+2.447, p<0.05) \\
\text { Virgin lands }(\mathrm{B}+\mathrm{SE}=3.562+1.769, p<0.05) \\
\text { Training during last } 5 \text { years }(\mathrm{B}+\mathrm{SE}=3.510+1.719, p<0.05) \\
\text { The farmer was advised to grow organic cotton }(\mathrm{B}+\mathrm{SE}=3.242+1.582, p<0.05) \\
\text { The ease in the use of the technology is an important aspect in the choice }(\mathrm{B}+\mathrm{SE}=4.295+1.746, p \\
\quad \text { The profitability of the technology is an important aspect in the choice }(\mathrm{B}+\mathrm{SE}=2.355+1.156, p< \\
\text { - } 0.05) \\
\text { Uses of synthetic fertilizers }(\mathrm{B}+\mathrm{SE}=-5.853+2.173, p<0.01) \\
\text { The decision of } \mathrm{GPC} \text { is an important aspect }(\mathrm{B}+\mathrm{SE}=-5.393+2.506, p<0.05)\end{array}$ \\
\hline 46 & $\begin{array}{l}\text { Ashari Sharifuddin et al. (2018) } \\
\text { [51] }\end{array}$ & Indonesia $^{a}$ & $\begin{array}{l}600 \\
\bullet \quad 300 \text { semi-organic } \\
-\quad 300 \text { conventional }\end{array}$ & $\begin{array}{ll}- & \text { Attitude }(\mathrm{B}=0.395, p<0.001) \\
- & \text { Perceived behavioral control }(\mathrm{B}=0.235, p<0.001) \\
- & \text { Moral obligation }(\mathrm{B}=0.125, p<0.01) \\
- & \text { Subjective norm }(\mathrm{B}=0.235, p<0.001)\end{array}$ \\
\hline 47 & Liu et al. (2019) [11] & USA $^{\text {a }}$ & $\begin{array}{l}456 \\
\text { (organic/conventional/mixed) }\end{array}$ & $\begin{array}{ll}- & \text { Operator age }(31-60 \text { vs. } 30 \text { or less })(\mathrm{B}+\mathrm{SE}=-2.45+1.01, p<0.05) \\
\text { - } & \text { Operator age }(61 \text { or above vs. } 30 \text { or less })(\mathrm{B}+\mathrm{SE}=-2.73+1.10, p<0.05) \\
\text { - } & \text { Attitude towards "Need exemplar to follow" }(\mathrm{B}+\mathrm{SE}=-2.01+0.44, p<0.001) \\
\text { - } & \text { Attitude towards "Low organic yield as barrier" }(\mathrm{B}+\mathrm{SE}=-0.95+0.43, p<0.05) \\
\text { - } & \text { Farm size }(\mathrm{B}+\mathrm{SE}=-2.48+0.69, p<0.001) \\
& \text { Farming years }(\mathrm{B}+\mathrm{SE}=-0.04+0.01, p<0.001) \\
& \text { Use farmers market }(\mathrm{B}+\mathrm{SE}=2.23+0.44, p<0.001)\end{array}$ \\
\hline
\end{tabular}


Table 1. Cont.

No. Authors (Year)

Country

\section{Findings (Factors Associated with Adoption of Organic Farming)}

Area of cold spots:

Level of natural productivity for organic farm $(\mathrm{B}+\mathrm{SE}=-0.135+0.016, p<0.01)$

- Organic operator is the agricultural production and marketing groups $(B+\mathrm{SE}=3.215+0.413$ $p<0.01)$

- $\quad$ Organic operator is individual farmers $(B+\mathrm{SE}=3.353+0.411, p<0.01)$

- Potential inundation of the organic farm $(\mathrm{B}+\mathrm{SE}=-3.976+0.349, p<0.01)$

- The closest distance to landslides from organic farm $(\mathrm{B}+\mathrm{SE}=-0.131+0.023, p<0.01)$

- The closest distance to the industrial zones from organic farm

- $\quad(\mathrm{B}+\mathrm{SE}=-0.060+0.003, p<0.01)$

- Area of hot spots:

- Organic operator is the agricultural production and marketing groups $(B+S E=1.227+0.273$ $p<0.01)$

- Organic operator is individual farmers $(\mathrm{B}+\mathrm{SE}=-2.761+0.297, p<0.01)$

- The issued year of the certificate in or after $(\mathrm{B}+\mathrm{SE}=-1.682+0.155, p<0.01)$

- Potential inundation of the organic farm $(\mathrm{B}+\mathrm{SE}=-2.507+0.611, p<0.01)$

- The closest distance to landslides from organic farm $(\mathrm{B}+\mathrm{SE}=0.365+0.027, p<0.01)$

The closest distance to the industrial zones from organic farm $(\mathrm{B}+\mathrm{SE}=0.035+0.005, p<0.01)$

- The closest distance to the industrial zones from org

- $\quad(\mathrm{B}+\mathrm{SE}=-3.269+1.349, p<0.05)$

Attitudes towards farming behavior $(r=0.263, p<0.05)$

- $\quad$ Perception of risk of farming $(\mathrm{r}=0.258, p<0.05)$

- Group-norm influence to farming behavior $(\mathrm{r}=0.254, p<0.05)$

- $\quad$ Support of government policy $(\mathrm{r}=0.135, p<0.05)$

- Comparative usefulness of behavior $(\mathrm{r}=0.332, p<0.05)$

- 448 organic

- 401 conventional

- Farming annual income $(\mathrm{r}=-0.128, p<0.05)$

- $\quad$ Attitude towards environment $(\mathrm{r}=0.256, p<0.01)$

- Attitude towards health $(r=0.246, p<0.01)$

- $\quad$ Attitude towards productivity and profit $(\mathrm{r}=0.241, p<0.01)$

- $\quad$ Subjective norms $(\mathrm{r}=0.184, p<0.01)$

- Perceive behavioral control $(\mathrm{r}=0.273, p<0.01)$

- $\quad$ Awareness of consequences $(\mathrm{r}=0.252, p<0.01)$

- $\quad$ Personal norms $(\mathrm{r}=0.280, p<0.01)$

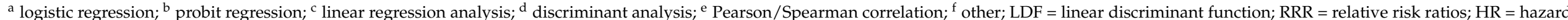
ratio; $\mathrm{B}=$ beta; $\mathrm{SE}=$ standard error; $\mathrm{r}=$ correlation coefficient. 
Table 2. Summary of the articles which describe the factors influencing farmers' adoption of organic farming $(n=50)$.

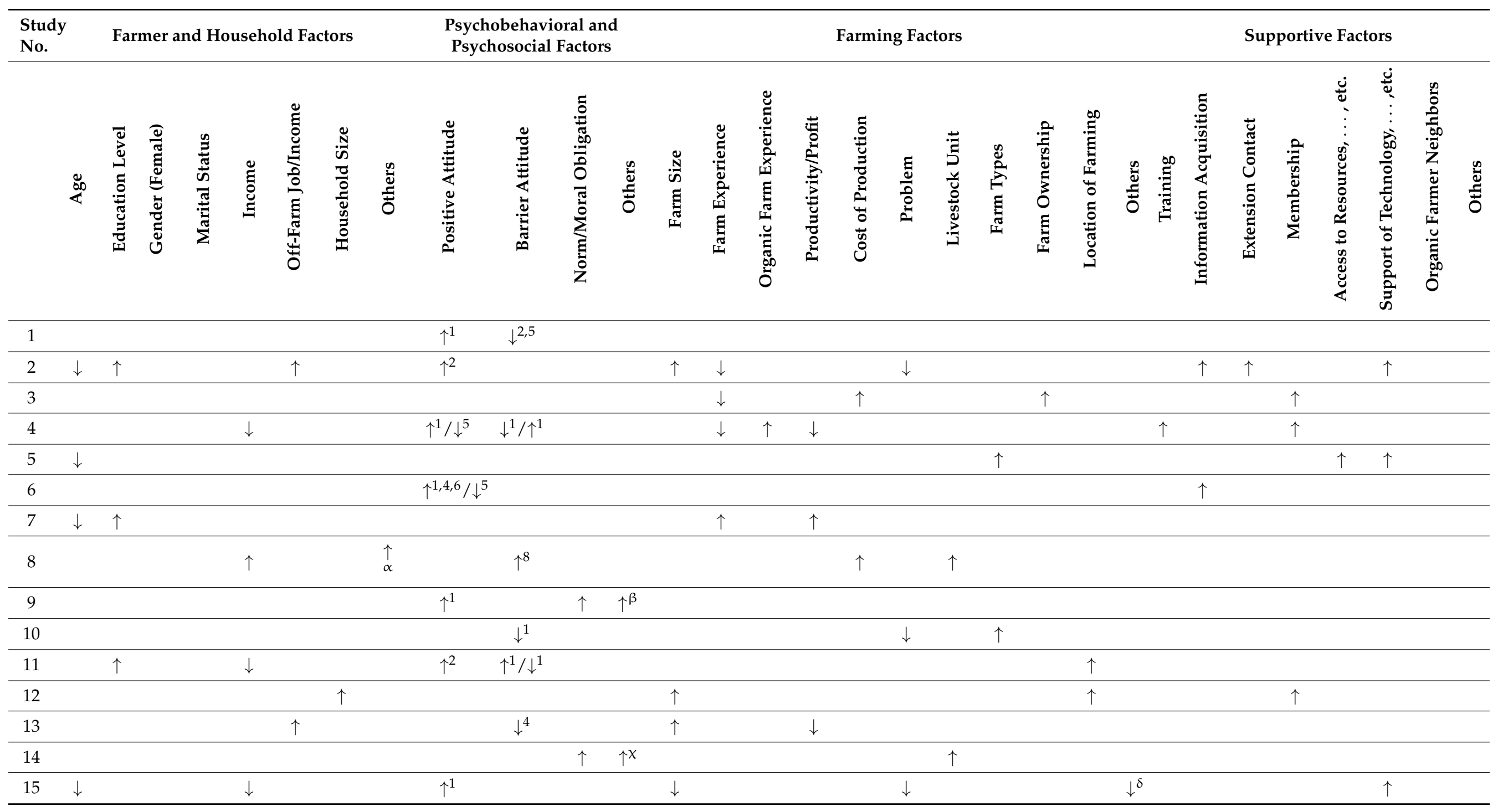


Table 2. Cont.

\begin{tabular}{|c|c|c|c|c|c|c|c|c|c|c|c|c|c|c|c|c|c|c|c|c|c|c|c|c|c|c|c|c|c|c|}
\hline \multicolumn{2}{|c|}{$\begin{array}{l}\text { Study } \\
\text { No. }\end{array}$} & \multicolumn{7}{|c|}{ Farmer and Household Factors } & \multicolumn{4}{|c|}{$\begin{array}{l}\text { Psychobehavioral and } \\
\text { Psychosocial Factors }\end{array}$} & \multicolumn{12}{|c|}{ Farming Factors } & \multicolumn{5}{|c|}{ Supportive Factors } & \multirow[b]{2}{*}{ } \\
\hline & $\stackrel{80}{4}$ & 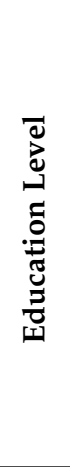 & 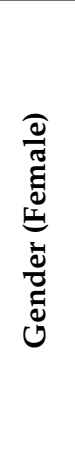 & 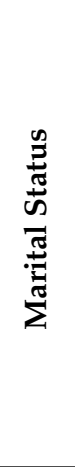 & 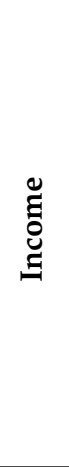 & 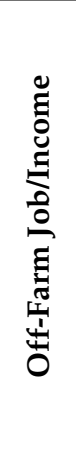 & 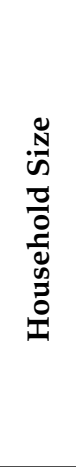 & & 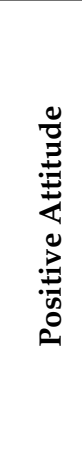 & 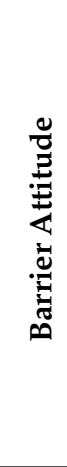 & 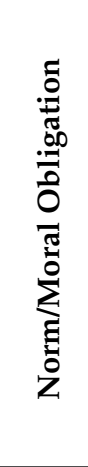 & & 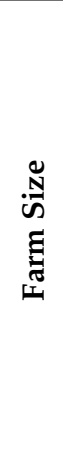 & 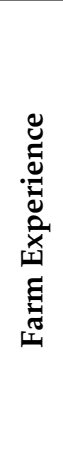 & 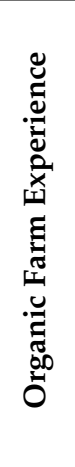 & 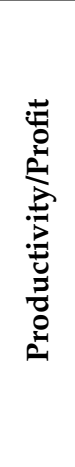 & 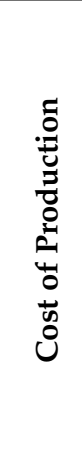 & 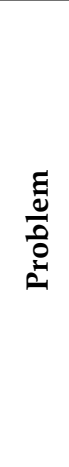 & 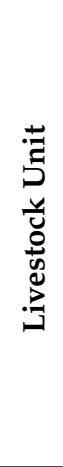 & 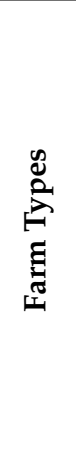 & 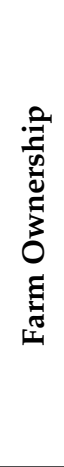 & 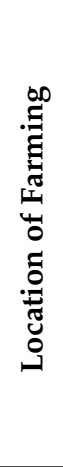 & 总 & 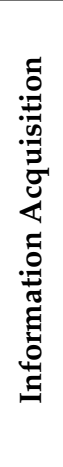 & 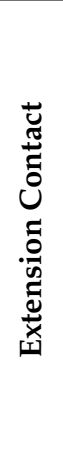 & 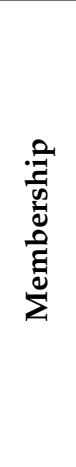 & 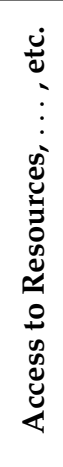 & 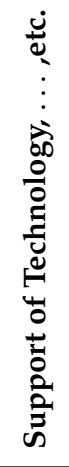 & 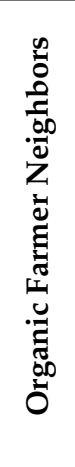 & \\
\hline 16 & & & & & & & & & $\uparrow^{2}$ & $\downarrow^{8}$ & & & $\downarrow$ & $\uparrow$ & & & $\uparrow$ & & $\downarrow$ & & & & & & & & & & $\uparrow$ & \\
\hline 17 & & & $\uparrow$ & & & & & & $\uparrow^{5}$ & & & & & & & & & $\downarrow$ & & & & & $\uparrow$ & & & & & $\uparrow$ & & \\
\hline 18 & $\uparrow$ & & & & & & & $\uparrow \varepsilon$ & & & & & $\uparrow$ & & & & & & & & & & $\uparrow \phi \uparrow$ & & & $\uparrow$ & & & & \\
\hline 20 & & & & & & & & & $\uparrow^{1,2}$ & & & & & & & & & & & $\uparrow$ & & & & $\uparrow$ & & & & & $\uparrow$ & \\
\hline 21 & $\downarrow$ & & & & & & $\downarrow$ & & $\uparrow^{2}$ & $\downarrow^{8}$ & & & $\downarrow$ & & & $\downarrow$ & & & $\downarrow$ & & & & & $\downarrow$ & & & & & $\uparrow$ & \\
\hline 22 & & & & & & & & & $\uparrow^{4} / \downarrow^{5}$ & & & & & & & & & & & & & & & & & & & & & \\
\hline 23 & & & & & & & & & $\uparrow^{1}$ & & & & & & & & $\uparrow$ & & & & & & & & & & $\uparrow$ & & & \\
\hline 24 & & $\uparrow$ & & & & & & & $\uparrow^{7}$ & & & & & & & & & & & & & & & & & & & & & \\
\hline 25 & $\downarrow$ & & & & & & & & & & & & & & $\uparrow$ & & & & & & & & & & & & $\downarrow$ & & & $\downarrow^{\eta}$ \\
\hline 26 & & $\uparrow$ & & & & & & & & & & $\uparrow \iota$ & $\downarrow$ & & & & & & $\uparrow$ & & & & $\uparrow \varphi \uparrow$ & & $\uparrow$ & & & & & \\
\hline 27 & & & & $\downarrow$ & & & & & & & & & & $\downarrow$ & & & & & & & & & & & & & & & & \\
\hline 28 & $\downarrow$ & & & & & & & & & & & & $\uparrow$ & $\uparrow$ & & & & & & & & & & & $\downarrow$ & & $\uparrow$ & & & \\
\hline 29 & & & & & & & & & $\uparrow^{1}$ & & & & & & & & & & & & & & & $\uparrow$ & & & & & & \\
\hline 30 & $\downarrow$ & & $\uparrow$ & & & & & & & & & & $\downarrow$ & & & $\uparrow$ & & & & $\downarrow$ & & $\uparrow$ & $\uparrow^{\kappa} / \downarrow^{\lambda}$ & & & & & $\uparrow$ & & \\
\hline
\end{tabular}


Table 2. Cont.

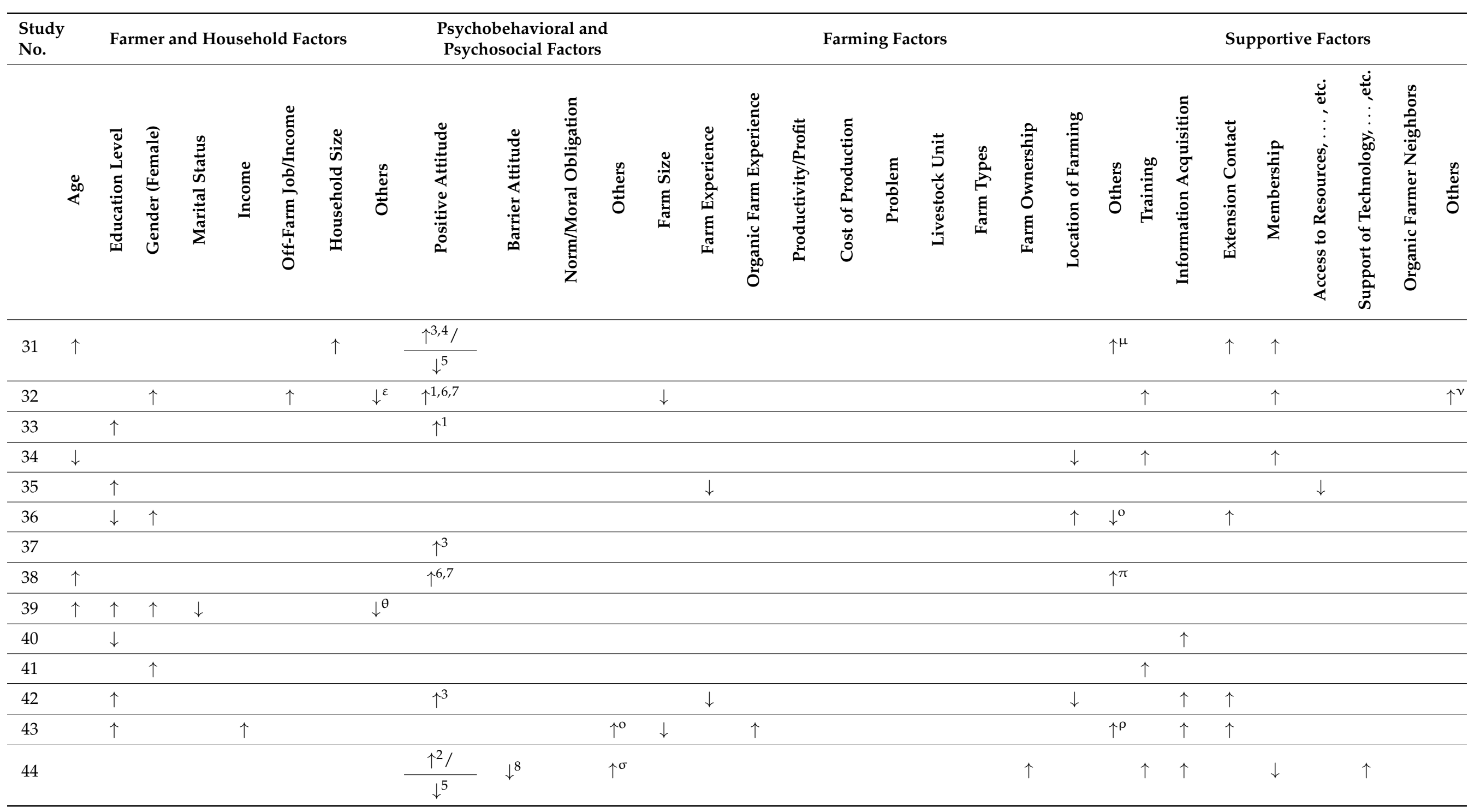


Table 2. Cont.

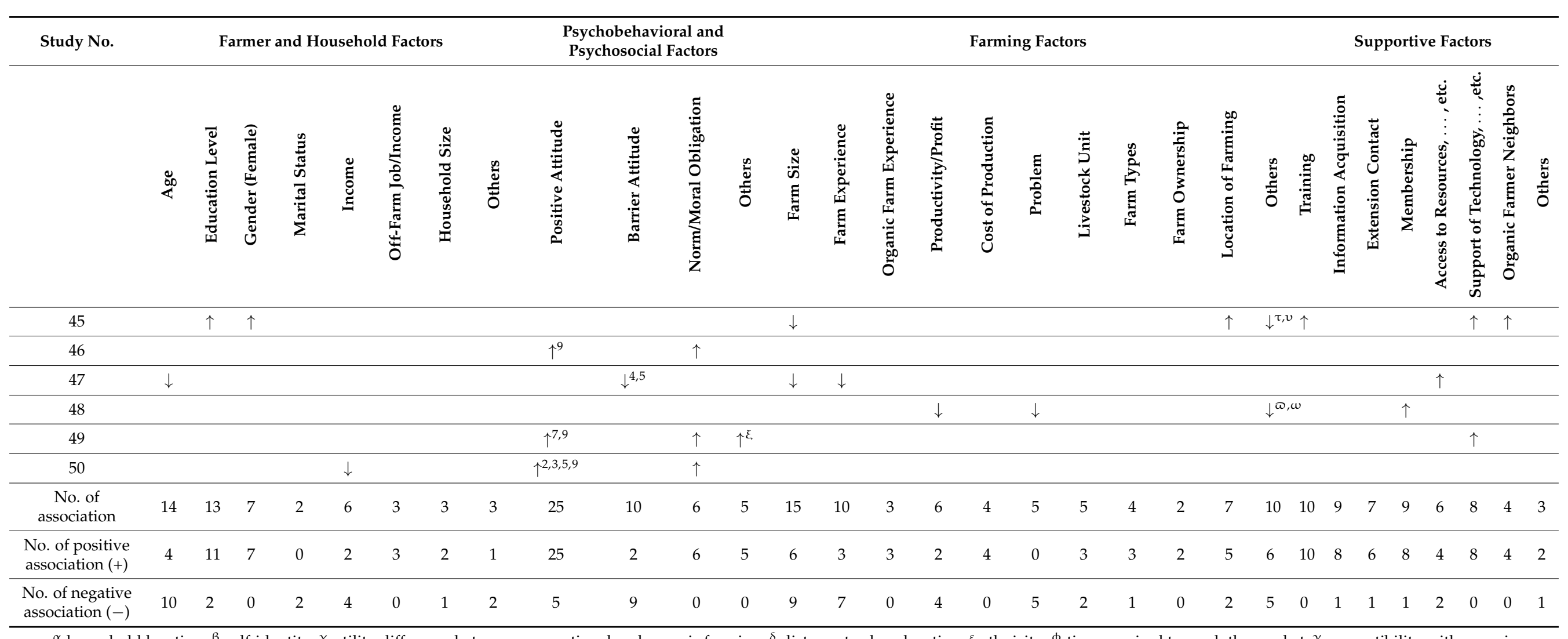

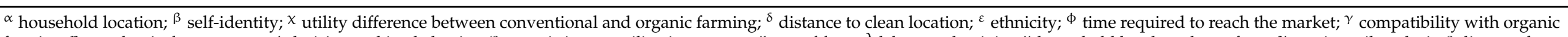

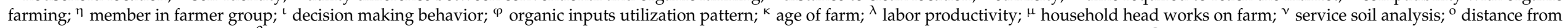

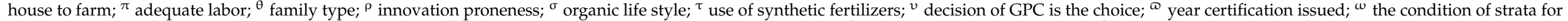

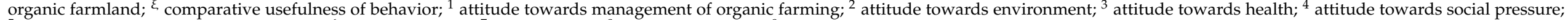
${ }^{5}$ attitude towards productivity and profit; ${ }^{6}$ perceived benefit; ${ }^{7}$ perceived risk; ${ }^{8}$ risk averse attitude; ${ }^{9}$ perceived behavioral control. 


\subsubsection{Age}

Fourteen articles found an association with age. Ten studies reported results indicating a negative association [9-17]. It is possible that younger farmers have more opportunities to access organic farming technology and minds more open to innovations than older farmers $[18,19]$. Another possibility is that older farmers are usually less educated and are uncertain about changing to organic farming $[9,20]$. Older farmers usually are by nature risk averse and have less time to invest for the long-term and expect future higher returns $[10,11]$. However, four studies showed that older farmers had more opportunities for adoption than younger famers [8,21-23]. This might be due to a good relationship with an extension service and more experience in farming [9,24]. However, the evidence available on age is still inconclusive.

\subsubsection{Education Level}

Thirteen articles found an association with education level, almost all of the studies reporting a positive association $[8,12,14,19,25-31]$, whereas only two articles found a negative association $[32,33]$. It is possible that educated farmers have a greater capacity to understand and realize the advantages of organic agriculture $[26,30]$. They also have more capacity to access organic farming information via several media resources [12].

\subsubsection{Gender}

Seven articles found an association with gender, and all available studies provided evidence that female farmers were more likely to adopt organic farming than male famers $[8,18,19,34-36]$. A possible explanation is that female farmers are particularly concerned about health effects of pesticides and about healthy food [32,34,36]. Nevertheless, organic farming by female farmers was often done on a small size with an off-farm income, whereas the main conventional farming was performed by male farmers [32,34].

\subsubsection{Marital Status}

Two articles pointed out that farmers who were married were negatively associated with adoption $[8,20]$.

\subsubsection{Income}

Six articles found an association with farmers' income; two articles a positive association [31,37], and four articles a negative association [15,25,38,39]. Additionally, KisakaLwayo [37] found that income from certified organic farmers was higher than for the other groups. This study suggested that a higher income made farmers able to rent more farmland, adopt new technology, and purchase necessary inputs for organic farming. A stable income plays a vital role in the conversion to organic farming. Income was also related to farm economy; therefore, farmers have to clearly understand comprehensive management $[25,31]$. Nevertheless, the evidence available is still inconsistent.

\subsubsection{Off-Farm Job or Income}

Three articles dealt with off-farm job/income, and all of the studies showed a similar association [12,24,35]. A study by Sriwichailamphan and Sucharidtham [35] suggested that if the farmers had extra income from off-farm sources, participation in organic farming would increase. The income from off-farm sources was the basis of for new technology adoption [12].

\subsubsection{Household Size}

Three articles found an association with household size, two articles reported a positive association [22,40], and a single article found a negative association [16]. Household size plays an important role in adoption regarding the number of family laborers. Family laborers are able to join in the activities on the farm, even if they work elsewhere, and consequently the household saves costs on hiring other workers [22,40,41]. In contrast, a 
study by Läpple and Rensburg [16] argued that the increase in household members was an obstacle to the making of free decisions.

\subsection{Psychobehavioral and Psychosocial Factors}

Psychobehavioral and psychosocial factors included a positive attitude to organic farming, barrier attitudes to organic farming, norms and moral obligations, and others.

\subsubsection{Positive Attitude, Perception, and Awareness of Organic Farming}

Twenty-five articles found an association with positive attitude. All available studies found a positive association, whereas five articles found both positive and negative associations at the same time $[12,15,16,22,23,25,26,28,30,34,35,38,39,41-52]$. Out of the 25 articles, attitudes can be categorized into seven aspects: attitude towards management of organic farming, attitude towards the environment, attitude towards health, attitude towards social influences, attitude towards profit and productivity, perceived benefits, and perceived risks.

The available literature showed that a positive attitude towards management of organic farming was the predominant factor. All available studies $(n=10)$ found a positive association $[15,28,35,38,42-44,46,48,49]$. Farmers thought that organic farming saved production costs, improved the marketing function, and facilitated effective management of the farm [15,35,46]. Kaufmann et al. [46] mentioned that farmers thought that they could manage organic farms effectively by themselves, due to information support from the government or other institutions. Similarly, all available studies $(n=6)$ found a positive association with regard to environmental attitude $[12,16,25,45,46,50]$. They thought that organic farming is better for the environment [46]. Koesling et al. [25] also mentioned that sustainable and environmentally friendly farming were the ultimate goal for organic farmers. Three articles found a positive association with attitudes to health $[22,30,41]$ and another three articles found a positive association with social attitude [22,43,47].

Perception is also a crucial determinant for adoption. Three articles found a positive association with perceived benefit $[23,35,43]$. Farmers perceived that organic farming was feasible on their farm and could increase their income. Similarly, two articles found a positive association with perceived behavioral control [51,52]. Regarding perceived risk, four articles found a positive association [23,26,35,52]. It is likely that farmers who have good risk perception prefer organic farming more than risk-averse farmers. An explanation is that less risk-averse farmers can bear the risk situation which include higher input costs, market price fluctuation, and market demand [23,52].

In contrast, six articles found a negative association as regards attitude towards profit and productivity $[22,34,38,43,47,50]$. This evidence implied that profit and productivity were not driving factors for adoption of organic farming [50].

\subsubsection{Barrier Attitudes to Organic Farming}

Ten articles found an association with barrier attitudes to organic farming [11,16,24, $25,37,38,42,45,50,53]$. Almost all of the articles $(n=9)$ found a negative association with barrier attitudes. The evidence implied that risk-averse farmers are less likely to adopt organic farming [16,37,45,50]. Läpple and Rensburg [16] also suggested that followers and laggard farmers were less likely to adopt organic farming than pioneer farmers, due to a weak potential to deal with risk situations. Farmers who thought that organic farming was unsuitable and complicated to practice were less likely to adopt organic farming [25]. Furthermore, farmers who had a positive attitude towards agrichemicals and pesticides were less likely to adopt organic farming [25,38].

\subsubsection{Norms and Moral Obligation}

Five articles, all those available, found a positive association with norms and moral obligation [39,44,51,52]. Ashari Sharifuddin et al. [51] revealed that farmers' family and influential people could influence the farmers in practicing organic farming. It is possible 
that farmers worry and feel the need to protect their family's health. Group norms also influenced farming behavior regarding the practice of organic farming [52].

\subsection{Farming Factors}

Farming factors included farm size, farm experience, organic farm experience, productivity/profit, cost of production, problems, livestock units, farm types, farm ownership, location, and others.

\subsubsection{Farm Size}

Fifteen articles found an association with farm size; nine articles found a negative association $[11,15,16,18,19,27,31,35,45]$, whereas six articles found a positive association [10, $12,21,24,40,54]$. It is possible that larger farms are more difficult to manage as regards crops, inputs, and other supports, resulting in farmers having less motivation to cope with these problems $[11,31,45]$. Furthermore, organic farming needs more intensive labor for the handling of pests, disease problems, and marketing. Therefore, organic farming is more likely to be adopted in small farms which are managed using family labors [16,45]. In contrast, some of the literature argued that large farms are more likely to adopt organic production. Farm size can imply wealth and social honorableness in an agricultural community. Therefore, farmers who have more land might have the capacity to use some parts of their land for the adoption of organic farming. Another explanation is that small farms are unable to access credit, resulting in the decline of adoption $[10,40]$. The evidence available on farm size is inconclusive.

\subsubsection{Farm Experience and Organic Farm Experience}

Ten articles found an association with farm experience. Seven articles found a negative association $[12,20,29,30,38,41,55]$, and three articles a positive one $[10,14,41]$. Farmers with longer experience are usually older and less educated, therefore it is difficult to shift them to the relatively new concept of organic farming $[11,20]$. Organic producers are usually newer entrants to the industry; therefore, the government and other institutions should encourage young farmers to incorporate organic agricultural practices $[38,55]$. Nevertheless, some of the literature argued that farmers with more experience in agricultural work are more likely to adopt organic farming, possibly due to their more extensive knowledge and their concern about environmental quality and the long-term impact [10,45]. Again, the evidence available is too inconsistent to formulate a verifiable conclusion.

With regard to experience in organic farming, two articles found a positive association $[17,38]$. It is probable that the farmers who have experience in organic farming realize the advantages of organic farming as regards health, productivity, markets, and environment [31].

\subsubsection{Productivity and Profit}

Six articles found an association with productivity and profit. Four articles found a negative association $[16,24,38,56]$, whereas two articles found a positive association $[14,18]$. Most available evidence showed that motivation from profit and productivity is not a favorable factor for farmers to engage in or shift towards organic farming, due to lack of markets, no subsidy support, and less income $[16,24,56]$. On the other hand, some of the literature mentioned that organic farming increased productivity and return on costs. Therefore, the evidence available is still too inconsistent for a firm conclusion.

\subsubsection{Cost of Production}

Four articles found a positive association with cost of production [37,45,48,55]. Organic farming might increase single farm payments, farm-gate price, input costs, and total costs $[37,55]$. These factors have a significant effect on the decision to engage in organic production. 


\subsubsection{Problems on Farms}

The literature mentioned that problems which were barriers to adopting organic farming practices included climatic conditions, inundation and landslides, pests, certification systems, and marketing problems [12,15,34,53,56]. A study by Lu and Cheng [56] suggested that potential inundation by and distance to landslide and industrial zones were negatively associated with adoption. Similarly, a study by Genius et al. [12] suggested that aridity indices were negatively associated with adoption, therefore unfavorable climatic conditions were a significant factor in the decision making process of farmers. With regard to marketing problems, a study by Khaledi et al. [15] found that if there was an increase in organic marketing problems with one unit, complete adoption of organic farming would decrease by approximately $19 \%$. Furthermore, a study by Thapa and Rattanasuteerakul [34] also suggested that farmers declined to adopt organic farming when they had to cope with serious pest problems. The evidence implies that farmers are less likely to adopt organic farming when they face uncontrollable problems, especially disasters.

\subsubsection{Livestock Unit}

Five articles found an association with livestock units; however, the associations were inconsistent [16,27,37,45,57]. The studies by Kisaka-Lwaya [37] and Best [57] found a correlation between higher livestock units and higher adoption. On the other hand, the studies by Läpple [45] and Läpple and Rensburg [16] found that higher livestock units meant lower adoption.

\subsubsection{Farm Types}

Four articles found an association with farm types, and almost all of the articles found a positive association $[13,18,46,53]$. A study by Anderson et al. [13] mentioned that the total number of crops grown on the farm was positively associated with adoption. Similarly, a study by Kaufmann et al. [46] stated that farmers who grew arable crops were more likely to convert to organic farming than farmers who adopted mixed farming (organic and non-organic). Likewise, a study by Canavari et al. [53] stated that mixed farmers were more likely to adopt organic farming than conventional farmers. The explanation for this could be that mixed farmers had management skills and positive attitudes towards production.

\subsubsection{Farm Ownership}

Two articles found a positive association with farm ownership [50,55], probably due to economic and social status [50].

\subsubsection{Location of Farms}

Seven articles found an association with location $[9,18,19,25,30,32,40]$. It is possible that the climatic and geographical situation of the farm might be more suitable for organic production. A study by Silas [40] suggested that farms in an eco-zone were more likely to adopt organic agriculture, due to the ready market for organic products, good community network, and high support from government.

\subsection{Supportive Factors}

Supportive factors are important determinants for adoption, which include training, information acquisition, contact with extension agents, membership of an association, access to resources and markets, technology support, motivation/subsidy, organic farmer neighbors, and others.

\subsubsection{Training}

Ten articles found a positive association with training [9,19,21,27,34-36,38,50,54]. Participation in training helps farmers to increase their knowledge through formal and informal education, and apply the information in their own field [27]. In addition, they can share knowledge and experience with their colleagues and neighbors [36,50]. A study 
by Singh et al. [9] also pointed out that participation in training more than once would increase organic farming by $13 \%$ and decrease inorganic farming by $11 \%$. Training programs run by academics, non-governmental or government organizations help farmers to learn cultivation practices and proper techniques for organic production. The program also informs farmers about the health and financial benefits of organic production $[9,34,35]$.

\subsubsection{Information Acquisition}

Nine articles found an association with information acquisition, and almost all of the articles $(n=8)$ found a positive association [12,16,30,31,33,43,46,49]. Studies by Savari et al. [49] and Pradhan et al. [31] suggested that use of educational publications and mass media were significant factors regarding adoption. Furthermore, a study by Kaufmann et al. [46] suggested that the number of agricultural information sources used was a significant factor for adoption of organic practices.

\subsubsection{Contact with Extension Agents}

Seven articles found an association with extension contacts [10,12,22,27,30-32]. Extension agents are important sources of information with regard to new agricultural technology and innovation; therefore, guidance from extension agents improves competence in utilizing new technology and innovation [27,32]. A study by Genius et al. [12] mentioned that farmers who sought agriculture information via extension agents had a higher probability of shifting to organic methods than farmers who did not seek such information. Similarly, a study by Ma et al. [30] reports that contact with extension agents increased those willing to adopt organic agriculture by $11.2 \%$. Likewise, studies by Sodjinou et al. [32] and Wolli and Andersson [13] also mentioned that farmers who participated in extension activities on more topics and more often were also more likely to adopt organic farming. Therefore, all the available studies provide strong evidence that interaction between extension agents and farmers are important in encouraging organic methods.

\subsubsection{Membership of Farming Association}

Nine articles found an association with membership of a farming association; eight articles found a positive association $[9,21,22,35,38,40,55,56]$, whereas only one article found a negative association [50]. Almost all of the articles implied that farmers who belonged to a farming association were more likely to adopt organic agriculture. The possible explanation for this is that activities in groups and knowledge shared by other organic farmers help the farmers to access information easily and solve problems regarding group certification and group marketing $[21,22,40]$. In addition, membership of a farming association can increase bargaining power with commission merchants [21].

\subsubsection{Access to Resources, Credits and Markets}

Two articles showed that access to a natural water supply contributed significantly to adoption of organic production $[29,48]$. With regard to access to marketing, two articles also revealed that access to marketing significantly contributed to farmers' adoption [11,13]. A study by Anderson et al. [13] suggested that farmers who used direct marketing strategies had a more than seven times higher adoption rate of organic agriculture. A study by Liu et al. [11] also suggested that farmers who used a farmers market for selling their products had an increased probability of approximately $26 \%$ of converting to organic farming. With regard to access to credit, a study by Rana et al. [10] mentioned that access to credit was a decision driver for the adoption of organic farming. In contrast, a study by Saoke [17] claimed that access to credit was a barrier to adoption, meaning that farmers who had the ability to access credit facilities were less likely to adopt organic farming.

\subsubsection{Support of Technology, Subsidy, and other Motivation}

Three articles found that technological support was a determinant for adoption $[13,15$, 19]. A study by Anderson et al. [13] pointed out that farmers who used computers for their 
farm tasks were 17 times more likely to adopt mixed farming rather than conventional farming. Likewise, a study by Khaledi et al. [15] pointed out that complete adoption of internet use for marketing organic products (33\%) was higher than for partial adopters $(13 \%)$, and internet use was positively associated with adoption of organic farming. With regard to subsidy, two articles found a positive association with subsidy $[12,18]$. Concerning motivation from other institutions, two articles found an association with motivation from the government, non-government, and business sectors [34,50,52]. Therefore, motivation programs have significant impact when it comes to the persuasion of farmers towards adoption.

\subsubsection{Organic Farming Neighbors}

Four articles revealed that farmers who know other organic farmers were more likely to adopt organic agriculture $[16,19,45]$. Social influences are important in the agricultural community. Organic farmers are an important source of relevant information when it comes to sharing their experience and persuading other farmers to adopt organic farming [45,46].

Figure 2 shows the percentage (\%) of articles which found a positive association with the adoption of organic farming for each factor. The factors which were covered with a positive association by more than $80 \%$ of articles were concluded as relevant to influencing the adoption of organic farming. The relevant factors for farmer and household factors included gender (female) $(100 \%)$, off-farm income $(100 \%)$, and education level $(84.6 \%)$. The relevant psychobehavioral and psychosocial factors included a positive attitude towards organic farming $(100 \%)$ and norms and moral obligation $(100 \%)$. The relevant farming factors included organic farming experience $(100 \%)$, cost of production $(100 \%)$, and farm ownership $(100 \%)$. The relevant supportive factors included training $(100 \%)$, support of technology, subsidy, and motivation $(100 \%)$, organic farmer neighbors $(100 \%)$, information acquisition $(88.9 \%)$, membership of an association $(88.9 \%)$, and contact with extension agents $(85.7 \%)$. Nevertheless, there are some limitations of this review. The levels of organic farming in each study are slightly different. Some studies investigated only complete organic farming, whereas some studies investigated partial or mixed organic farming. Therefore, the interpretation of factors may have been affected. 


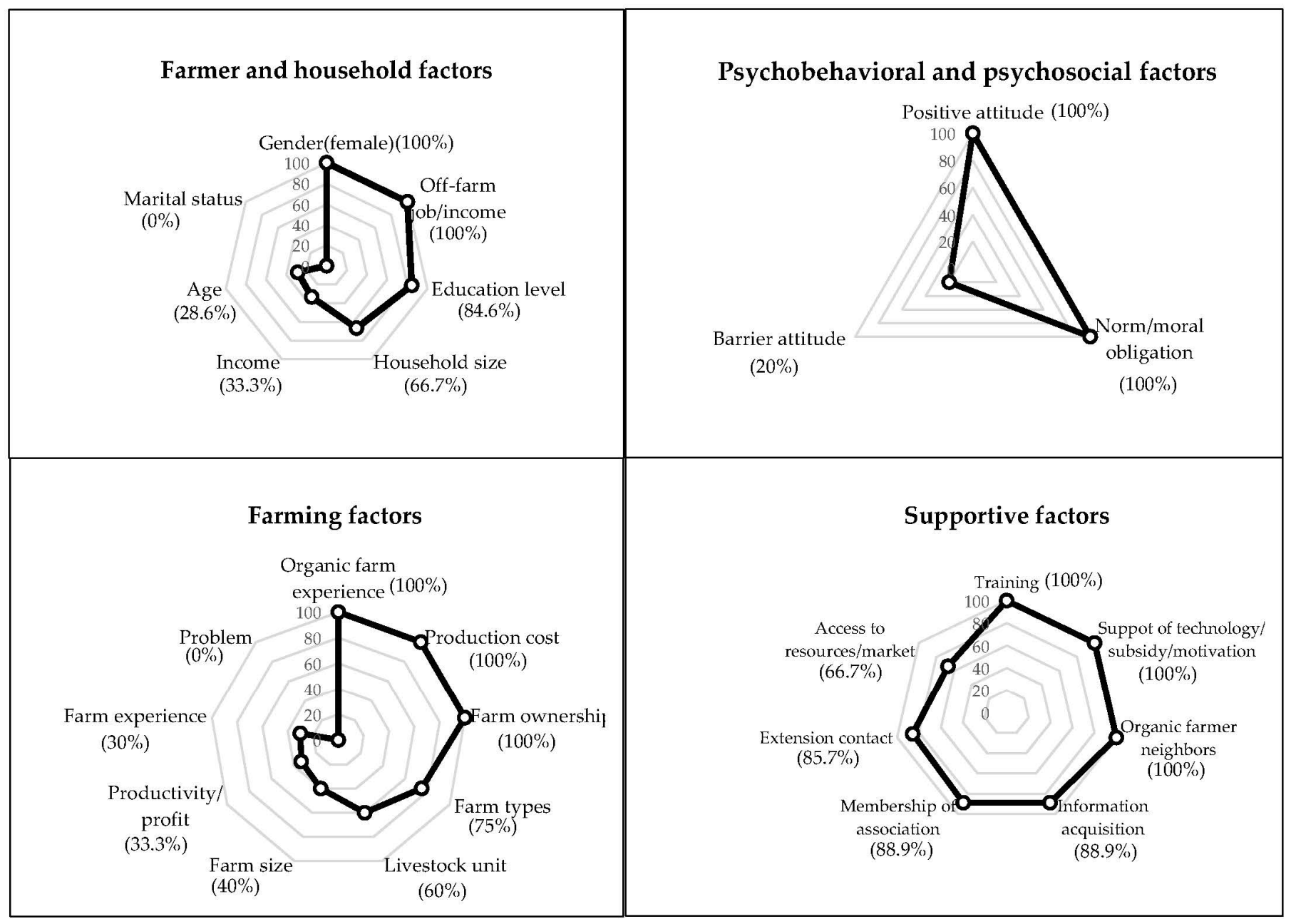

Figure 2. Percentage (\%) of articles which found a positive association with adoption of organic farming.

\section{Conclusions}

All the relevant available studies provided evidence that the significant factors influencing farmers' adoption of organic farming are as follows: (1) farmer and household factors (i.e., gender, and off-farm income) (2) psychobehavioral and psychosocial factors (i.e., positive attitude to organic farming, norms and moral obligation); (3) farming factors (i.e., organic farm experience, cost of production, and farm ownership); and (4) supportive factors (i.e., training, support of technology/subsidy/motivation, and organic farmer neighbors).

To promote and expand the adoption of organic farming, training is most important to give knowledge, information, and technology about organic farming to farmers. Therefore, extension agents play a vital role in the continuous arrangement of training programs. The main issues which need addressing in training programs included advantages of organic farming, cultivation practices, proper care of organic production, and technology and innovation adoption. Furthermore, extension agents also play a vital role in creating a positive attitude to organic farming and encouraging conventional farmers to shift towards organic agriculture. The target groups that are potentially more likely to adopt organic farming are young farmers, females, farm owners, those with a high level of education, and those with an off-farm income. Membership of a farm association is also a major determinant for adoption of organic farming. Members can share information and experience with their colleagues and increase their bargaining power with commission merchants. Membership of a farm association also encourages a positive attitude towards organic farming and norms in the agricultural community. The literature also identifies 
that support from the government as regards resources, credit, markets, and subsidy is relevant in motivating the adoption of organic farming. The policy of the government should support organic farmers in having easy access to water supplies for agriculture, and access to credits and markets. Government policy should reinforce the function of the extension services to change the perception and behavior of pesticide handlers sustainably. The government needs to support technology and subsidy to farmers who adopt organic farming. Therefore, the three sectors, extension agents, farm associations, and the government, are key drivers for promoting the sustainable adoption of organic farming.

Author Contributions: Conceptualization: R.S.; Methodology: R.S.; data analysis: R.S., A.T.; Writing-Original draft preparation: R.S.; Writing-Review and Editing: R.S.; Funding acquisition: R.S. All authors have read and agreed to the published version of the manuscript.

Funding: This study was supported by the Thailand Science Research and Innovation Fund, Thailand (Grant No. RSA6280030).

Institutional Review Board Statement: The Human Ethical Committee at Faculty of Medicine, Chiang Mai University (No. EXEMPTION 7274/2020).

Informed Consent Statement: Not applicable.

Data Availability Statement: Not applicable.

Acknowledgments: We would like to thank the Thailand Science Research and Innovation Fund, Thailand (Grant no. RSA6280030).

Conflicts of Interest: All of the authors declare that they have no competing interests.

\section{References}

1. Nicolopoulou-Stamati, P.; Maipas, S.; Kotampasi, C.; Stamatis, P.; Hens, L. Chemical pesticides and human health: The urgent need for a new concept in agriculture. Front. Public Health 2016, 4. [CrossRef] [PubMed]

2. Damalas, C.A.; Koutroubas, S.D. Farmers' exposure to pesticides: Toxicity types and ways of prevention. Toxics 2016, 4. [CrossRef] [PubMed]

3. Lee, K.S.; Choe, Y.C.; Park, S.H. Measuring the environmental effects of organic farming: A meta-analysis of structural variables in empirical research. J. Environ. Manag. 2015, 162, 263-274. [CrossRef]

4. Mishra, P.; Singh, P.P.; Singh, S.K.; Verma, H. Sustainable agriculture and benefits of organic farming to special emphasis on PGPR. In Role of Plant Growth Promoting Microorganisms in Sustainable Agriculture and Nanotechnology; Woodhead Publishing: Cambridge, UK, 2019; pp. 75-87. [CrossRef]

5. IFOAM. Organics International The World of Organic Agriculture 2019. Available online: https://www.ifoam.bio/en/news/20 19/02/13/world-organic-agriculture-2019 (accessed on 13 April 2020).

6. Smith, S.; Paladino, A. Eating clean and green? Investigating consumer motivations towards the purchase of organic food. Australas. Mark. J. 2010, 18, 93-104. [CrossRef]

7. Nguyen, H.V.; Nguyen, N.; Nguyen, B.K.; Lobo, A.; Vu, P.A. Organic food purchases in an emerging market: The influence of consumers' personal factors and green marketing practices of food stores. Int. J. Environ. Res. Public Health 2019, 16, 1037. [CrossRef] [PubMed]

8. Azam, M.; Banumathi, M. The role of demographic factors in adopting organic farming: A logistic model approach. Int. J. Adv. Res. 2015, 3, 713-720. Available online: https:/ / www.journalijar.com/article/5847/the-role-of-demographic-factors-in-adoptingorganic-farming:-a-logistic-model-approach/ (accessed on 12 November 2020).

9. Singh, M.; Maharjan, K.L.; Maskey, B. Factors impacting adoption of organic farming in chitwan district of Nepal. Asian Econ. Soc. Soci. 2015, 5, 1-12. [CrossRef]

10. Rana, S.; Parvathi, P.; Waibel, H. Factors affecting the adoption of organic pepper farming in India. In Proceedings of the International Research on Food Security, Natural Resource Management and Rural Development, Göttingen, Germany, 19-21 September 2012; Available online: http:/ / www.ifgb.uni-hannover.de/fileadmin/eagr/EUE_files/Publicat_Download/2012 /tropentag-abstract.pdf (accessed on 15 December 2020).

11. Liu, X.; Pattanaik, N.; Nelson, M.; Ibrahim, M. The choice to go organic: Evidence from small US farms. Agric. Sci. 2019, 10, 1566-1580. [CrossRef]

12. Genius, M.; Pantzios, C.; Tzouvelekas, V. Information acquisition and adoption of organic farming practices: Evidence from farm pperations in Crete, Greece. J. Agric. Resour. Econ. 2006, 31, 93-113. [CrossRef] 
13. Anderson, J.; Jolly, D.; Green, R. Determinants of farmer adoption of organic production methods in the fresh-market produce sector in California: A logistic regression analysis. In 2005 Western Agricultural Economics Association Annual Meeting. 2005. Available online: https://ideas.repec.org/p/ags/waeasa/36319.html (accessed on 15 December 2020).

14. Isin, F.; Tayfun, C.; Goksel, A. Factors affecting the adoption of the drganic dried fig Agriculture system in Turkey. J. Appl. Sci. 2007, 7, 748-754. [CrossRef]

15. Khaledi, M.; Weseen, S.; Sawyer, E.; Ferguson, S.; Gray, R. Factors influencing partial and complete adoption of organic farming practices in Saskatchewan, Canada. Can. J. Agric. Econ. 2010, 58, 37-56. [CrossRef]

16. Läpple, D.; van Rensburg, T. Adoption of organic farming: Are there differences between early and late adoption? Ecol. Econ. 2011, 70, 1406-1414. [CrossRef]

17. Saoke, L.A. Organic Farming in the Kibera Slum in Nairobi, Kenya. Master's Thesis, Wageningen University, Wageningen, The Netherlands, 2011. Available online: https:/ /library.wur.nl/WebQuery/theses/2006232 (accessed on 7 December 2020).

18. Malá, Z.; Malý, M. The determinants of adopting organic farming practices: A case study in the Czech Republic. Agric. Econ. 2013, 59, 19-28. [CrossRef]

19. Métouolé Méda, Y.J.; Egyir, I.; Zahonogo, P.; Jatoe, J.; Atewamba, C. Institutional factors and farmers' adoption of conventional, organic and genetically modified cotton in Burkina Faso. Int. J. Agric. Sustain. 2018, 16, 40-53. [CrossRef]

20. Adesope, O.; Matthews-Njoku, E.C.; Nkasiobi, O.; Ugwuja, V.C. Effect of socio-economic characteristics of farmers on their adoption of organic farming practices. Crop Prod. Technol. 2012, 211-220. [CrossRef]

21. Karki, L.; Schleenbecker, R.; Hamm, U. Factors influencing a conversion to organic farming in Nepalese tea farms. J. Agric. Rural Dev. Trop. 2011, 112, 113-123. Available online: https://core.ac.uk/download/pdf/146490546.pdf (accessed on 15 December 2020).

22. Wollni, M.; Andersson, C. Spatial patterns of organic agriculture adoption: Evidence from Honduras. Ecol. Econ. 2014, 97, 120-128. [CrossRef]

23. Xie, Y.; Zhao, H.; Pawlak, K.; Gao, Y. The development of organic agriculture in China and the factors affecting organic farming. J. Agribus. Rural Dev. 2015, 2, 353-361. [CrossRef]

24. Alexopoulos, G.; Koutsouris, A.; Tzouramani, I. Should I stay or should I go? factors affecting farmers' decision to convert to organic farming or to abandon it. In Proceedings of the 9th European IFSA Symposium, Vienna, Austria, 4-7 July 2010; Available online: http:/ /ifsa.boku.ac.at/cms/fileadmin/Proceeding2010/2010_WS2.3_Alexopouos.pdf (accessed on 12 November 2020).

25. Koesling, M.; Flaten, O.; Lien, G. Factors influencing the conversion to organic farming in Norway. Int. J. Agric. Resour. Gov. Ecol. 2008, 7, 78-95. [CrossRef]

26. Radwan, A.; Gil, J.; Diab, Y.; Abo-Nahoul, M. Determinants of the adaption of organic agriculture in Egypt using a duration analysis technique. In Proceedings of the 85th Annual Conference, Coventry, UK, 18-20 April 2011. [CrossRef]

27. Prashanth, P.; Reddy, M.M. Factors influencing the adoption of organic farming by the farmers of Karimnagar district of Andhra Pradesh. Int. J. Farm Sci. 2012, 2, 123-128. [CrossRef]

28. Shaban, A. Factors influencing farmers' decision to shift to organic farming: The case of Gaza Strip. J. Econ. Manag. Trade 2015, 5, 78-87. [CrossRef]

29. Pinthukas, N. Farmers' perception and adaptation in organic vegetable production for sustainable livelihood in Chiang Mai Province. Agric. Agric. Sci. Procedia 2015, 5, 46-51. [CrossRef]

30. Ma, W.; Ma, C.; Su, Y.; Nie, Z. Organic farming: Does acquisition of the farming information influence Chinese apple farmers' willingness to adopt? China Agric. Econ. Rev. 2017, 9, 211-224. [CrossRef]

31. Pradhan, M.; Tripura, B.; Mondal, T.K.; Darnnel, R.R.; Murasing, J. Factors influencing the adoption of organic farming by the farmers of North District of Sikkim. Int. J. Adv. Sci. Res. Dev. 2017, 4, 1-7. Available online: http:/ / paper.researchbib.com/view / paper $/ 200707$ (accessed on 10 November 2020).

32. Sodjinou, E.; Glin, L.; Nicolay, G.; Tovignan, S.; Hinvi, J. Socioeconomic determinants of organic cotton adoption in Benin, West Africa. Agric. Food Econ. 2015, 3, 1-12. [CrossRef]

33. Cukur, T. Conventional dairy farmers converting to Ooganic dairy production in Turkey. Pol. J. Environ. Stud. 2015, $24,1543-1551$ [CrossRef]

34. Thapa, G.; Rattanasuteerakul, K. Adoption and extent of organic vegetable farming in Mahasarakham province, Thailand. Appl. Geograph. 2011, 31, 201-209. [CrossRef]

35. Sriwichailamphan, T.; Sucharidtham, T. Factors affecting adoption of vegetable growing using organic system: A case study of Royal Project Foundation, Thailand. Int. J. Econ. Manag. Sci. 2014, 3, 1000179. [CrossRef]

36. Kerdsriserm, C.; Suwanmaneepong, S.; Mankeb, P.; Factors affecting adoption of organic rice farming in sustainable agriculture network, Chachoengsao Province, Thailand. Int. J. Agric. Technol. 2016, 12, 1227-1237. Available online: http:/ / www.ijataatsea. com/pdf/v12_n7_1_16_DecemberSpecialissue/Aricultural\%2012no7.1\%20(1229-1240)\%20(1)\%201.pdf (accessed on 15 December 2020).

37. Kisaka-Lwayo, M. A discriminant analysis of factors associated with the adoption of certified organic farming by smallholder farmers in Kwazulu-Natal, South Africa. In Proceedings of the 2007 Second International Conference, Accra, Ghana, 20-22 August 2007.

38. Parra López, C.; Calatrava Requena, J. Factors related to the adoption of organic farming in Spanish olive orchards. Span. J. Agric. Res. 2005, 3, 5-16. [CrossRef] 
39. Nguyen, T.; Nguyen, T.; Doan, X.; Tran, M.; Tran, M.; Nguyen, T. A dataset of factors influencing intensions for organic farming in Vietnam. Data Brief 2020, 33, 106605. [CrossRef]

40. Peter Silas, M. Factors Affecting Adoption of Organic Farming by MAIZE Farmers in MERU South District. Master' s Thesis, Kenyatta University, Kenyatta, Kenya, 2008. Available online: https:/ /ir-library.ku.ac.ke/handle/123456789/8672 (accessed on 15 December 2020).

41. Ullah, A.; Shah, S.N.M.; Ali, A.; Naz, R.; Mahar, D.A.; Kalhoro, S. Factors affecting the adoption of organic farming in PeshawarPakistan. Agric. Sci. 2015, 6, 587-593. [CrossRef]

42. Egri, C.P. Attitudes, backgrounds and information preferences of canadian organic and conventional farmers: Implications for organic farming advocacy and extension. J. Sustain. Agric. 1999, 13, 45-72. [CrossRef]

43. De Cock, L. Determinants of Organic Farming Conversion. In Proceedings of the The 11 International Congress of the European Association of Agricultural Economists (EAAE), Copenhagen, Denmark, 23-27 August 2005. [CrossRef]

44. McCarthy, M.; Reilly, S.; O'Sullivan, A.; Guerin, P. An investigation into the determinants of commitment to organic farming in Ireland. J. Farm Manag. 2007, 13, 135-152. Available online: http:/ /ifmaonline.org/wp-content/uploads/2014/08/07McCarthy_ etal_2.pdf (accessed on 12 November 2020).

45. Läpple, D. Adoption and abandonment of organic farming: An empirical investigation of the Irish drystock sector. J. Agric. Econ. 2010, 61, 697-714. [CrossRef]

46. Kaufmann, P.; Zemeckis, R.; Skulskis, V.; Kairyte, E.; Stagl, S. The Diffusion of Organic Farming in Lithuania. J. Sustain. Agric. 2011, 35, 522-549. [CrossRef]

47. Mzoughi, N. Farmers adoption of integrated crop protection and organic farming: Do moral and social concerns matter? Ecol. Econ. 2011, 70, 1536-1545. [CrossRef]

48. Pornpratansombat, P.; Bauer, B.; Boland, H. The adoption of organic rice farming in northeastern Thailand. J. Org. Sys. 2011, 6,1-12. Available online: https://www.organic-systems.org/journal/Vol_6(3)/pdf/JOS_6(3)_2011_04-12_Pornpratansombat.pdf (accessed on 10 November 2020).

49. Savari, M.; Ebrahimi-Maymand, R.; Mohammadi-Kanigolzar, F. The Factors influencing the application of organic farming operations by farmers in Iran. Econ. Inform. 2013, 5, 179-187. [CrossRef]

50. Haris, N.B.M.; Garrod, G.; Gkartzios, M.; Proctor, A. The Decision to Adopt Organic Practices in Malaysia: A Mix-method Approach. In Proceedings of the 92 Annual Conference, Coventry, UK, 16-18 April 2018. [CrossRef]

51. Ashari Sharifuddin, J.; Mohamed, Z.; Terano, R.; Sharifuddin, J. Paddy farmer's perception and factors influencing attitude and intention on adoption of organic rice farming. Int. Food Res. J. 2018, 25, S135-S145. Available online: http:/ / www.ifrj.upm.edu. my /25\%20(08)\%202018\%20supplementary\%202/3\%20-\%20IFRJ18255.R2\%20edited.pdf (accessed on 12 November 2020).

52. Yanakittkul, P.; Aungvaravong, C. A model of farmers intentions towards organic farming: A case study on rice farming in Thailand. Heliyon 2020, 6, e3039. [CrossRef] [PubMed]

53. Canavari, M.; Cantore, N.; Lombardi, D. Factors explaining farmers' behaviours and intentions about agricultural methods of production. Organic vs. conventional comparison. In Proceedings of the 16th IFOAM Organic World Congress, Modena, Italy, 16-20 June 2008; Available online: https:/ / orgprints.org/id/file/57274 (accessed on 12 November 2020).

54. Kafle, B. Factors affecting adoption of organic vegetable farming in Chitwan District, Nepal. World J. Agric. Sci. 2011, 7, 604-606. Available online: https: / / citeseerx.ist.psu.edu/viewdoc/download?doi=10.1.1.415.5266\&rep=rep1\&type=pdf (accessed on 10 November 2020).

55. Hattam, C.; Holloway, G. Adoption of Certified Organic Production: Evidence from Mexico. In Proceedings of the International Scientific Conference on Organic Agriculture "Researching Sustainable Systems", Adelaide, Australia, 21-23 September 2005; Available online: http:/ / orgprints.org/4367/ (accessed on 15 December 2020).

56. Lu, C.F.; Cheng, C.Y. Impacts of spatial clusters on certified organic farming in Taiwan. Sustainability 2019, 11, 2637. [CrossRef]

57. Best, H. Environmental cocern and the adoption of organic agriculture. Soc. Nat. Resour. 2010, 23, 451-468. [CrossRef] 